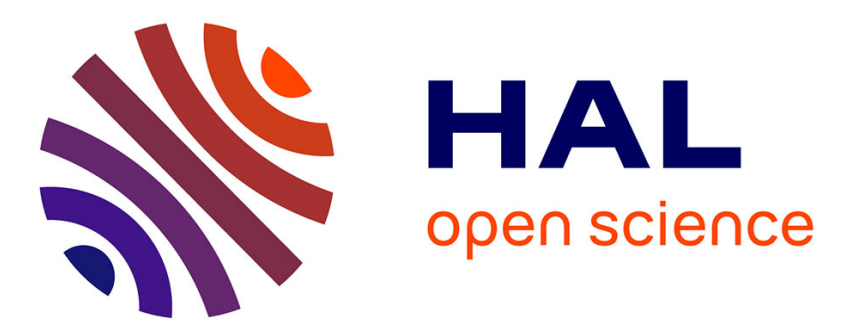

\title{
Plasma properties conditioned by the magnetic throat location in a helicon plasma device
}

Alfio E Vinci, Stéphane Mazouffre

\section{To cite this version:}

Alfio E Vinci, Stéphane Mazouffre. Plasma properties conditioned by the magnetic throat location in a helicon plasma device. Journal of Applied Physics, 2021, 130 (18), pp.183301. 10.1063/5.0069983 . hal-03474101

\section{HAL Id: hal-03474101 \\ https://hal.science/hal-03474101}

Submitted on 10 Dec 2021

HAL is a multi-disciplinary open access archive for the deposit and dissemination of scientific research documents, whether they are published or not. The documents may come from teaching and research institutions in France or abroad, or from public or private research centers.
L'archive ouverte pluridisciplinaire HAL, est destinée au dépôt et à la diffusion de documents scientifiques de niveau recherche, publiés ou non, émanant des établissements d'enseignement et de recherche français ou étrangers, des laboratoires publics ou privés. 


\title{
Plasma properties conditioned by the magnetic throat location in a Helicon plasma device
}

\author{
Alfio $\mathrm{E}$ Vinci $^{\mathrm{a})}$ and Stéphane Mazouffre ${ }^{\mathrm{b})}$ \\ Institut de Combustion, Aérothermique, Réactivité et Environnement (ICARE), Centre National de la Recherche Scientifique, \\ 1C Avenue de la Recherche Scientifique, 45071 Orléans, France
}

(Dated: 21 September 2021)

\begin{abstract}
Measurements are conducted in a Helicon plasma device to analyze the spatial distribution of plasma properties as the throat of the magnetic nozzle is axially shifted with respect to the antenna center. Krypton plasma is generated in the sub-kilowatt range and probed using a suite of diagnostics including a rf-compensated Langmuir probe, a planar probe and laser-induced fluorescence. It is found that larger ion currents and increased plasma confinement are achieved when the throat of the magnetic nozzle is located downstream the antenna center, at a distance that equals or exceeds two times the antenna length. The ions, although being accelerated, retain subsonic velocities even beyond the magnetic throat.
\end{abstract}

\section{I. INTRODUCTION}

8 A recent keen interest in electric space propulsion stems 9 from an increasing number of small satellites being deployed 10 for plural applications ${ }^{1-6}$. Simple and compact thrusters can ${ }_{11}$ allow for in-space maneuvering of such small spacecrafts ${ }^{7}$ 12 which usually do not boast large volume capacity to meet the 13 needs of consolidated technologies, e.g. Gridded Ion thrusters 14 and Hall thrusters. As a result, a portion of research and devel${ }_{15}$ opment efforts are being focused on electrodeless devices and 16 magnetic nozzle $(\mathrm{MN})$ acceleration ${ }^{8-13}$. This kind of technol17 ogy can indeed offer a number of advantages compared to the 18 aforementioned state-of-the-art devices. Issues linked to ero19 sion of components are expected to be of little consequence 20 due to the absence of direct interaction between plasma dis${ }_{21}$ charge and electrodes, resulting in a long lifetime. The plasma 22 beam leaving the discharge chamber is quasineutral, thus no ${ }_{23}$ neutralizer is required. Furthermore, these plasma sources 24 can more easily operate using condensible propellants with 25 respect to those system that require a dedicated neutralizer, ${ }_{26}$ whose lifetime and reliable functioning can be considerably 27 affected $^{14}$.

${ }_{28}$ The Helicon plasma (HP) thruster is one such a device. It 29 mainly comprises three components: $i$ ) a dielectric tube as so plasma discharge region, $i i$ ) a radiofrequency (rf) antenna for 31 gas ionization and iii) an axially directed steady magnetic field 32 for plasma confinement and expansion throughout its diverg33 ing region, i.e. the magnetic nozzle. Power radiated by the an34 tenna is deposited in the electron population as thermal energy 35 by means of helicon wave modes ${ }^{15}$ and Trivelpiece-Gould ${ }_{36}$ wave modes ${ }^{16}$. The energy is used for plasma production and 37 transport phenomena, such as ambipolar electric field, result${ }_{38}$ ing in axial acceleration of the ions in the $\mathrm{MN}$ by conversion 39 of the electron thermal energy.

40 The maturity of this technology is still relatively low ${ }^{5}$, ${ }_{41}$ whereby several undesired aspects come along with the con42 sidered advantages. The most critical one perhaps regards the

a)alfio.vinci@cnrs-orleans.fr

b) stephane.mazouffre@cnrs-orleans.fr
${ }_{43}$ thrust efficiency. Thus far, direct thrust measurements have ${ }_{44}$ revealed efficiencies typically below $10 \%$ in many different 45 devices ${ }^{13,17-24}$, with the only recent exception of nearly $20 \%$ ${ }_{46}$ at several $\mathrm{kW}$ of input power ${ }^{12}$. In this scenario, improv${ }_{47}$ ing the attained performance is required in order to turn HP 48 thrusters into a competitive technology in the near future. The 49 better understanding of the physics governing such rf plasma 50 sources by exploring the different working parameters is cur${ }_{51}$ rently the preferred approach to provide an exhaustive opti52 mization pathway.

53 In an effort to model HP discharges, previous analy54 ses have considered different magnetic topologies inside 55 the source tube, e.g. uniform ${ }^{25,26}$, Helmholtz and Maxwell ${ }_{56}$ configurations $^{27}$. The magnetic field inside the source is then 57 matched with a divergent geometry through a magnetic throat 58 localized in the proximity of the tube exit. This firm condi59 tion is not necessarily the most convenient solution, as demon${ }_{60}$ strated in Ref. 28 where a stepped-diameter tube is employed ${ }_{61}$ and the magnetic throat is placed downstream the exit cross ${ }_{62}$ section of the small main tube. Besides, virtually every nu${ }_{63}$ merical and experimental work studying the influence of mag${ }_{64}$ netic nozzle on the plasma properties, limited the analysis to 65 the exploitation of different magnitudes at the throat. A fruit${ }_{66}$ ful investigation is reported in Ref. 29, where the location of ${ }_{67}$ the magnetic throat is indirectly shifted with respect to the an${ }_{68}$ tenna - which is constantly located at the back plate - by ${ }_{69}$ changing the discharge tube length. Another relevant analy70 sis is reported in Ref. 30, where the authors characterized the ${ }_{71}$ plasma and the rf power circuit when the magnetic throat lo${ }_{72}$ calized either within the source or at the exhaust. Bennet et ${ }_{73} \mathrm{al}^{31}$ studied the generation of plasma as a function of the MN 74 throat location. Yet, their setup did not include plasma expan75 sion outside the discharge tube and many operating conditions 76 relied on magnetized ions, which is not a typical scenario in 77 propulsion. The shape of the magnetic nozzle and the location 78 of the throat are presumably strongly linked to the amount 79 of the input energy that can be usefully converted into axial 80 thrust. Energetic electrons are mainly produced close to the ${ }_{81}$ region occupied by the antenna. Several phenomena occur ${ }_{82}$ in the nozzle, such as transport due to pressure gradient and 83 electron cooling via convection and/or conduction, which de${ }_{84}$ termine the conversion efficiency of inlet thermal energy into 


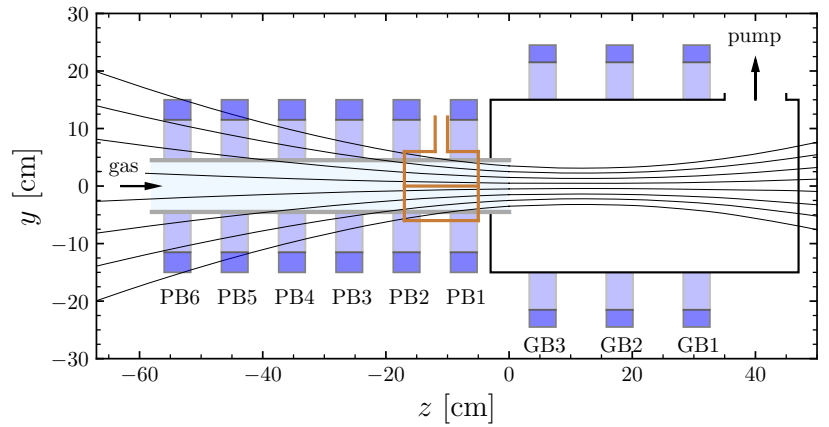

FIG. 1. Helicon source schematics. The $z=0$ position refers to the discharge tube outlet, $y=0$ refers to the reactor axis.

${ }_{85}$ ion acceleration. Furthermore, as the throat is shifted along ${ }_{86}$ the thruster axis with respect to the antenna location, the ex${ }_{87}$ pansion ratio of the plasma beam changes, thus resulting in ${ }_{88}$ possibly different thrust levels.

${ }_{89}$ This paper reports on experimental results obtained in a HP 90 source operating with krypton under several configurations of ${ }_{91}$ the external magnetic field. Numerous plasma properties are 92 spatially resolved as the throat of the magnetic field is moved ${ }_{93}$ with respect to the antenna axial position. A detailed descrip94 tion of the experimental setup is provided in Section II, in95 cluding the HP source and the magnetic configurations. In ${ }_{96}$ Section III, the diagnostics are extensively described. They in97 clude: a rf-compensated Langmuir probe to spatially charac98 terize plasma density, electron temperature, and plasma poten99 tial; a planar probe to measure the ion current density; laser100 induced fluoresce to explore the axial ions and atoms velocity 101 distribution function. Measurements are presented and dis${ }_{102}$ cussed in Section IV. Eventually, conclusions are drawn in ${ }_{103}$ Section V.

\section{II. EXPERIMENTAL SETUP}

105 A schematics of the HP source employed in this study is 106 shown in Figure 1 along with an example of computed mag${ }_{107}$ netic field lines. The entire experimental setup is oriented in a 108 vertical position.

109 The discharge chamber consists in a borosilicate glass tube 110 with $\phi=9 \mathrm{~cm}$ inner diameter and $L=58 \mathrm{~cm}$ length, whose 111 open exit arbitrarily identifies the origin of the axial coordi112 nate $z$. Krypton gas is introduced off-axis in the tube through ${ }_{113}$ its top aperture at a nominal flow rate of $1 \pm 0.004 \mathrm{mg} \mathrm{s}^{-1}$. ${ }_{114}$ The bottom part is connected to a $30 \mathrm{~cm}$ in inner diame115 ter and $50 \mathrm{~cm}$ in length expansion chamber made in alu116 minum which is equipped with a turbomolecular/primary 117 pumping system. The ultimate base pressure is in the order 118 of $10^{-5}$ mbar, whereas the typical pressure during operation ${ }_{119}$ reads $\sim 5 \times 10^{-3}$ mbar. ${ }_{120}$ The rf antenna in use is a double-saddle type with ${ }_{146}$ ing the ideal shape of the plasma plume (whether it is assumed ${ }_{121} d_{A}=12 \mathrm{~cm}$ length centered at $z \cong-11 \mathrm{~cm}$. It is made of ${ }_{147}$ fully magnetized). With reference to Figure 2 , it is noteworthy 122 pure copper and features two feeding arms which are long 148 to emphasize on the magnetic throat location. It moves from ${ }_{123}$ enough to be directly connected to the matching network so 149 upstream the antenna in Conf. A to far downstream the an-
FIG. 2. 2D profiles of the externally applied magnetic fields. White line indicates the discharge tube walls, white rectangles represent the magnetic coils, the gray rectangle indicates the antenna region and tube edge at $z=0$.

124 that power losses due to intermediate links are prevented. In125 put $\mathrm{rf}$ power is generated by a $1 \mathrm{~kW}$-class power supply at $12613.56 \mathrm{MHz}$ and kept constant to $750 \mathrm{~W}$ throughout the re${ }_{127}$ ported measurements. The power carried by higher order har${ }_{128}$ monics is certified to be lower than $-40 \mathrm{dBc}$. Matching of 129 the rf power is attained via a custom $\pi$-type network. During 130 operation, values of the reflected power are below $\sim 2 \%$.

A set of nine electromagnets surrounds both the discharge 132 tube and the expansion chamber. Their denomination is re133 ported in Figure 1. Each electromagnet is made up of a $2 \mathrm{~mm}$ 134 diameter copper wire, constituting 510 loops for PB1-6 and 135430 loops for GB1-3. The presence of multiple electromag136 nets enables the investigation of profoundly different mag137 netic field topologies. For the purpose of this study, five mag138 netic configurations are examined by powering two electro139 magnets at once. In doing so, the location of the magnetic 140 throat is intentionally relocated along the $z$ axis. The tested 141 magnetic configurations are progressively labeled as Conf. A 142 to Conf. E. When involved, PB coils are powered with $3 \mathrm{~A}$ 143 and GB coils with $6 \mathrm{~A}$ of direct current. Figure 2 shows the ${ }_{144}$ computed 2D magnetic field maps, highlighting the stream-

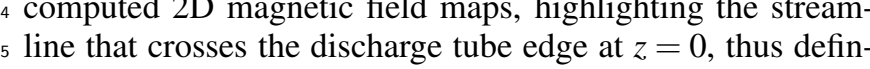
the black solid line exemplifies the magnetic streamline crossing the 


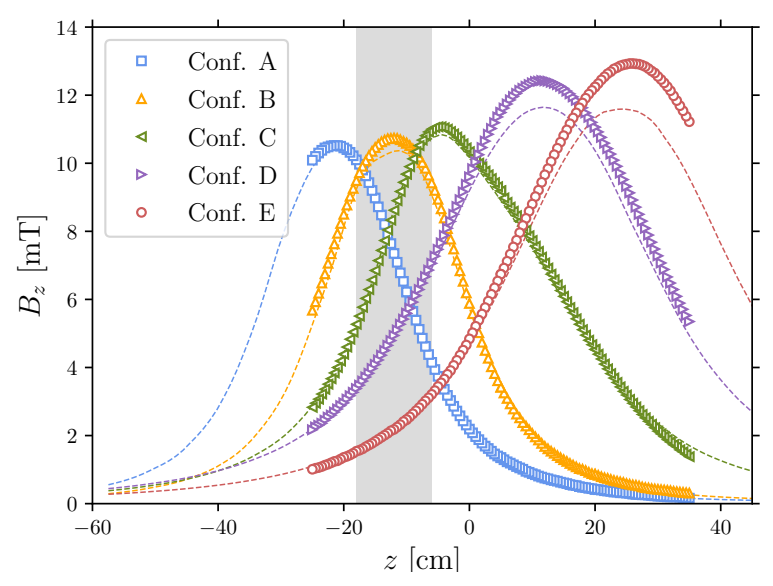

FIG. 3. Externally applied magnetic field on-axis profiles. Shaded area indicates the antenna location. Dashed lines represent simulation profiles, scatter points indicate probe measurements.

150 tenna in Conf. E. The first axial mode the antenna can generate 151 translates in an axial wavelength as $\lambda_{z}=24 \mathrm{~cm}$, see Section ${ }_{152}$ IV C. Notice that the magnetic throat in Conf. D is approxi153 mately one wavelength downstream of the antenna center. The 154 magnitude of the on-axis magnetic field is reported in Figure 1553 by comparing computed values and measured values. All 156 magnetic configurations feature a peak value of $10 \div 13 \mathrm{mT}$ ${ }_{157}$ with a similar qualitative profile but shifted along $z$.

\section{${ }_{158}$ III. DIAGNOSTICS}

\section{${ }_{159}$ A. RF-compensated Langmuir probe}

160 Plasma density $n_{p}$, electron temperature $T_{e}$ and plasma po${ }_{161}$ tential $V_{p}$ are measured using a rf-compensated Langmui ${ }_{162}$ probe (LP), see Figure 4. A detailed description of the probe ${ }_{163}$ has been previously reported in ${ }^{11}$. It is designed in accor${ }_{164}$ dance with the results reported $i^{32-34}$. A tungsten wire of ${ }_{165} 0.38 \mathrm{~mm}$ in diameter and $5 \mathrm{~mm}$ in length constitutes the probe 166 tip. An additional electrode made of stainless steel contributes ${ }_{167}$ to rf compensation and provides mechanical support to the as${ }_{168}$ sembly. The electrode is electrically insulated from the probe ${ }_{169}$ tip using a $1 \mathrm{~mm}$ in outer diameter alumina tube. A $1 \mathrm{nF}$ ax170 ial capacitor is soldered to the compensation electrode and 171 connected in parallel with the probe tip and a series of three 172 axial chokes, each of which self-resonates at one of the first 173 three harmonics. The whole assembly is encapsulated inside 174 a borosilicate glass tube of $6 \mathrm{~mm}$ outer diameter, which also 175 accommodates the coaxial cable for probe biasing and current

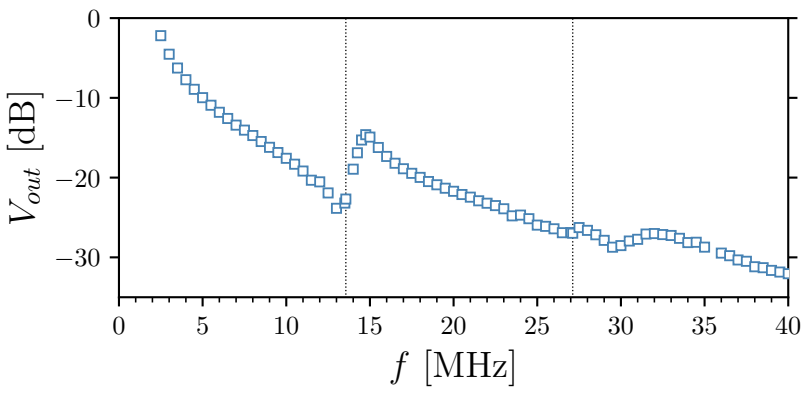

FIG. 5. RF-compensated LP frequency spectrum $\left(V_{\text {ref }}=10 \mathrm{~V}\right)$. Vertical dotted lines indicate $13.56 \mathrm{MHz}$ and $27.12 \mathrm{MHz}$.

176 measuring.

Chokes selection is attained by characterization of their 178 impedance frequency spectrum. A $20 \mathrm{~V}_{p p}$ sinewave is gener179 ated by a function generator and fed to one lead of the testing ${ }_{180}$ choke. The other lead is connected to a $46.52 \mathrm{k} \Omega$ resistor and 181 the series circuit closes to the ground. The frequency of the ${ }_{182}$ input sinewave is swept while the voltage drop across the re${ }_{183}$ sistor is monitored and used to calculate the current flowing 184 in the circuit. The frequency at which the current is minimum 185 is the choke self-resonating frequency. Functional check of 186 the whole probe assembly is performed by applying a $20 \mathrm{~V}_{p p}$ ${ }_{187}$ sinewave to the tungsten tip at different frequencies. The ${ }_{188}$ probe output is recorded by a digital oscilloscope and shown 189 in Figure 5 as a function of the input frequency. Local minima 190 are evident in correspondence of the harmonic frequencies, as 191 desired.

${ }_{192}$ The probe is manually displaced within the plasma using 193 a single-axis translation stage with a resolution of $10 \mu \mathrm{m}$ and 194 a stroke of $150 \mathrm{~mm}$. Probe alignment is ensured by the use 195 of a cross-line laser pendulum. I-V curves are recorded us196 ing a ALP control unit by Impedans Ltd. Thereafter, data is 197 post-processed relying on OML theory ${ }^{34,35}$. Assuming local 198 quasineutrality, plasma density $n_{p}$ is inferred from the linear 199 fit of the ion current squared versus the probe potential. The 200 ion current fit is then subtracted from the total current to ob201 tain a better estimation of the electron current. Assuming the 202 electrons are described by a Maxwell-Boltzmann distribution 203 function, the logarithmic profile of the electron current is lin204 early fitted, enabling the estimation of the electron tempera${ }_{205}$ ture $T_{e}$. The plasma potential $V_{p}$ is computed as the probe bias 206 voltage where the first derivative of the I-V curve features its 207 peak value.

Uncertainty associated with the probe measurements is es209 timated through a statistical approach. The detailed procedure 10 has been previously reported in ${ }^{11}$. The outline of the analysis ${ }_{211}$ shows that all plasma parameters are measured with a statisti${ }_{212}$ cal deviation as high as $\sim 2 \%$.

\section{B. Planar probe with guard ring}

214 The ion current density $j_{i}$ is measured using a planar probe ${ }_{215}$ with a guard ring (PPGR). It mainly comprises a disk-shaped 


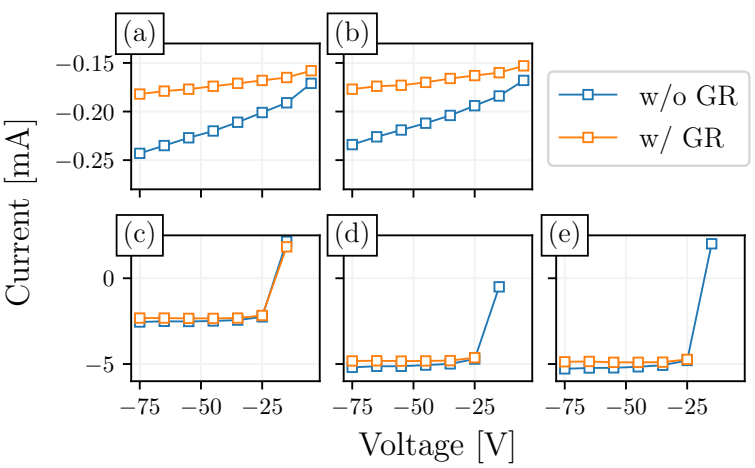

FIG. 6. Ion current profiles at $(x, y=0 ; z=15 \mathrm{~cm})$. Subplots denomination is linked to magnetic configurations Conf.s A-E.

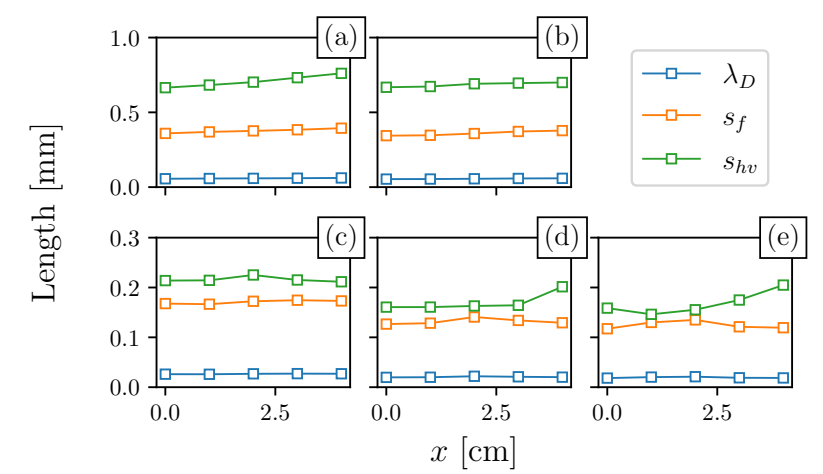

FIG. 7. Debye length, floating and high-voltage sheath thickness at $z=15 \mathrm{~cm}$. Subplots denomination is linked to magnetic configurations Conf.s A-E.

256 to perform a more qualitative-like comparison of the five mag257 netic configurations.

Given the high level of reproducibility recorded during pre25 liminary measurements, the uncertainty in $j_{i}$ is mainly given 260 by the uncertainty in the measured current and the probe col261 lecting area. This consideration leads to a value of $<8 \%$.

The planar probe is manually actuated from outside the 263 chamber using the single-axis translation stage described in 264 Section III A.

224 tial irregularities. The collector is $5.6 \mathrm{~mm}$ in diameter and ${ }_{225} 1 \mathrm{~mm}$ in thickness, whereas the ring width is $1 \mathrm{~mm}$. A gap 226 of $100 \mu \mathrm{m}$ is maintained between the two electrodes to ensure ${ }_{227}$ electrical insulation. Each electrode features a separated elec228 trical connection for polarization and current measurement. ${ }_{229}$ Voltage is applied to the collector by way of a Keithley 2410 230 SourceMeter, which is also used to measure the flowing cur231 rent. The guard ring is biased using a TTIEX752M power 232 supply.

${ }_{233}$ Functional check of the probe is carried out by recording 234 the $\mathrm{I}-\mathrm{V}$ curves at $(x, y=0 ; z=15 \mathrm{~cm})$ for the five magnetic 235 configurations denominated Conf.s A-E. The curves obtained 236 with a biased and floating guard ring are compared in Figure ${ }_{237}$ 6. Saturation of the ion current is achieved for Conf.s C-D-E ${ }_{238}$ at voltages lower than about $-40 \mathrm{~V}$, while no complete satu239 ration is recorded for Conf.s A and B due to the fact that the 240 guard ring width is not compatible with the local values of ${ }_{241}$ the sheath thickness. This can be confirmed by computing the 242 local values of the characteristic lengths over the radial coor${ }_{243}$ dinate using the values of $n_{p}$ and $T_{e}$ later discussed in Section $244 \mathrm{IV}$. Floating and high voltage sheath thickness, $s_{f}$ and $s_{h v}$ re${ }_{245}$ spectively, are computed in accordance with $^{37}$, using $-75 \mathrm{~V}$ 246 for Conf.s A-B and $-50 \mathrm{~V}$ for Conf.s C-D-E. Outcomes are ${ }_{247}$ plotted in Figure 7. It results evident that values of $s_{h v}$ for ${ }_{248}$ Conf.s C-D-E are much smaller than the guard ring width, 249 whereas those for Conf.s A and B approach its size. It shall 250 be noted that the attainment of a perfectly flat profile of the ${ }_{251}$ ion saturation current would require a guard ring width $5 \div 10$ 252 times larger than the actual one. Thus, the overall probe size 253 would probably lead to serious plasma perturbations. Further254 more, the scope of the presented measurements is not to, e.g., 255 focus on indirect propulsive performance estimation but rather

\section{Laser-induced fluorescence setup}

Ions and atoms velocity distribution functions (VDFs) are 67 measured by means of laser-induced fluorescence (LIF) spec268 troscopy. The technique and the optical bench in use have been extensively described in previous works ${ }^{38,39}$. Excita270 tion of metastable levels is achieved via an amplified tunable single-mode laser diode in the $810-840 \mathrm{~nm}$ spectral range. 272 The primary laser beam is split in multiple beams with the 23 purpose of $i$ ) instantaneous monitoring of the laser mode and 274 detection of any mode hop by means of a Fabry-Pérot interfer275 ometer, ii) laser power check using a photodiode and iii) accu276 rate wavelength measurement by way of a calibrated waveme277 ter whose absolute uncertainty is $\sim 60 \mathrm{~m} \mathrm{~s}^{-1}(80 \mathrm{MHz})$. Mod278 ulation of the laser beam is achieved using a mechanical chop279 per at $\sim 1.9 \mathrm{kHz}$. The laser is coupled to a $50 \mu \mathrm{m}$ optical 280 fiber which allows transporting the laser beam from the optical 281 bench to the Helicon source setup. Laser is injected inside the 282 vacuum chamber through a borosilicate glass window located

TABLE I. Kr I and Kr II probed optical transitions.

\begin{tabular}{llll}
\hline \hline & Transition & $\begin{array}{l}\lambda_{\text {exc }}[\mathrm{nm}] \\
\text { (vacuum) }\end{array}$ & $\begin{array}{l}\lambda_{\text {fluo }}[\mathrm{nm}] \\
\text { (air) }\end{array}$ \\
\hline $\mathrm{Kr}$ I & $5 s^{2}[3 / 2]_{1}^{\circ} \rightarrow 5 p^{2}[3 / 2]_{2}$ & 819.23095 & 760.15457 \\
$\mathrm{Kr}$ II & $5 p^{2} D_{5 / 2}^{\circ} \rightarrow 5 s^{2} P_{3 / 2}$ & 820.4959 & 461.91658 \\
\hline \hline
\end{tabular}




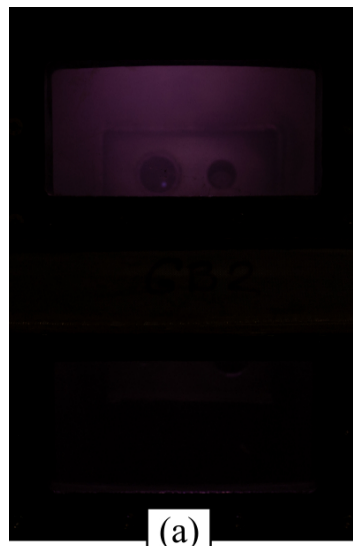

(a)

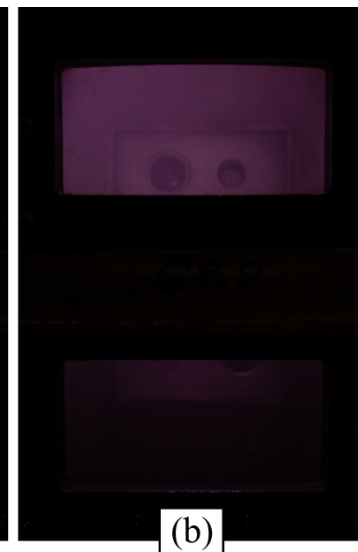

(b)
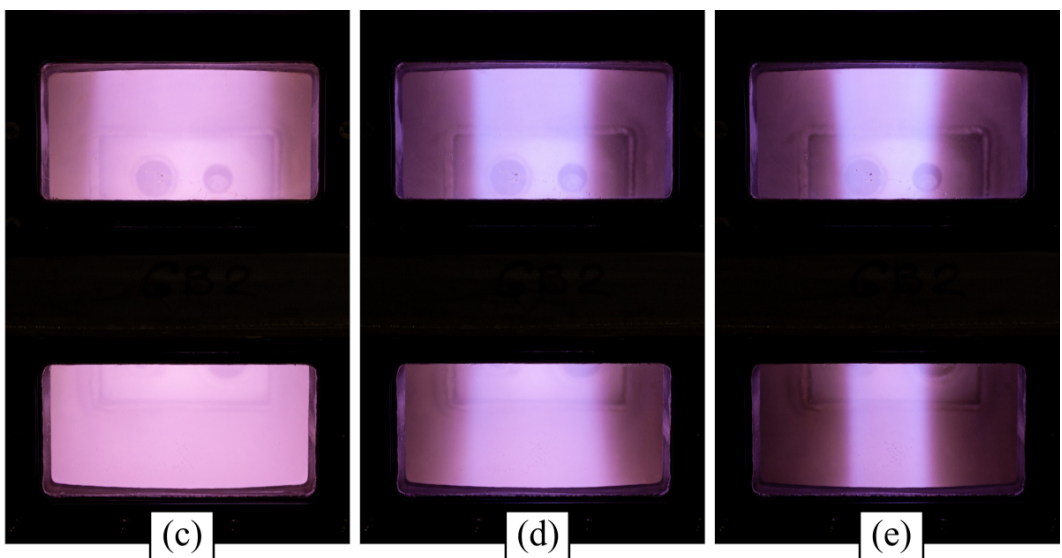

FIG. 8. Photographs of the plasma plume in $(a)$ Conf. A, $(b)$ Conf. B, $(c)$ Conf. C, $(d)$ Conf. D and $(e)$ Conf. E. Windows are centered at $z=12$ and $z=24 \mathrm{~cm}$.

283 at the bottom of the reactor. Note that $\mathbf{k} \cdot \mathbf{v}<0$ here, where $\mathbf{k}$ 284 is the laser wavenumber and $\mathbf{v}$ the particle velocity. The laser 285 beam is collimated using a combination of a $150 \mathrm{~mm}$ and a $2861000 \mathrm{~mm}$ focal length plano-convex lenses. Uncertainty on 287 the alignment with respect to the HP source axis is estimated 288 to be $\lesssim 1.6 \mathrm{deg}$. In order to ensure a linear regime of the 289 studied optical transitions, the laser power density delivered 290 to the plasma is kept below $1.2 \mathrm{~W} \mathrm{~mm}^{-2}$, a threshold value 291 that has been determined during preliminary measurements. 292 A detection branch is placed perpendicularly to the excitation 293 beam featuring a pair of plano-convex lenses $(250 \mathrm{~mm}$ and $294100 \mathrm{~mm}$ focal lengths) focusing on a $200 \mu \mathrm{m}$ core diameter 295 optical fiber. Both the excitation and detection branches are 296 mounted on a dedicated computer-controlled high precision 297 linear translation stage to enable spatially resolved measure298 ments along the $y$ axis. The detection optical train can be 299 additionally displaced along the $z$ axis within a few centime300 ters using a manual stage. The $200 \mu \mathrm{m}$ optical fiber transports 301 the fluorescence light to a monochromator which isolates the 302 desired line from the whole spectrum. Afterwards, a photo303 multiplier tube is used to convert the light signal into volt304 age, which is fed into a lock-in amplifier operating at the laser 305 modulation frequency to distinguish the signal from the nat306 ural plasma emission. The VDFs are inferred by scanning 307 over the laser wavelength $\lambda$ and computing the particles axial 308 velocity $v_{z}$ as $v_{z}=c\left(\lambda-\lambda_{0}\right) / \lambda_{0}$, where $\lambda_{0}$ is the unshifted 309 transition wavelength and $c$ is the speed of light. The mea310 sured LIF profiles result from the Doppler broaden line. The 311 presence of $\mathrm{Kr}$ isotopes leads to broadening due to isotopic 312 shift and hyperfine structure ${ }^{38}$. Moreover, due to the pres313 ence of a steady magnetic field, the Zeeman effect splits the 314 spectral lines into sub-levels ${ }^{38}$. Nevertheless, since the spec315 trum is dominated by the ${ }^{84} \mathrm{Kr}$ isotope and the magnetic field 316 is relatively weak, it is herein assumed that the observed fluo317 rescence line profile images the local VDF.

318 The probed optical transitions are reported in Table I. The 319 zero-velocity wavelength for Kr I is estimated from prelimi320 nary measurements in the HP source shining the laser along 321 the $x$ axis. The other listed wavelengths are given in the 322 literature ${ }^{38-42}$

\section{${ }_{323}$ IV. EXPERIMENTAL RESULTS AND DISCUSSION}

\section{A. Visual inspection}

25 The very first piece of information regarding the way the 326 plasma responds to the applied magnetic field topology is pro327 vided by visual inspection. The photographs of the plasma 328 plume reported in Figure 8 are taken using a common DSLR 329 camera framing the region $8<z<28 \mathrm{~cm}$ and set at $\mathrm{f} / 8$ and $3301 / 40$ s exposure time. It results evident how significant differ331 ences characterize the extracted plasma plume when employ332 ing the different magnetic topologies. A faint plume is visible 333 when the reactor operates in Conf. A and Conf. B. Instead, a 334 well defined plasma beam is noticed when Conf.s C, D and E 335 are chosen. In the latter cases, the plasma radius visibly delin336 eates the local magnetic streamlines, cf. Figure 2 and Figure8. 337 It is interesting to notice that the local magnitude of the ax338 ial component $B_{z}$ is nearly equal for Conf. $\mathrm{C}$ and Conf. $\mathrm{E}$ at з39 $z=12 \mathrm{~cm}$ (see Figure2), i.e. at the center of the top window 340 in Figure8. Yet, the two plasma beams show a very different 341 structure. As a matter of fact, local values of the field radial 342 component $B_{r}$ and gradient $\partial B_{z} / \partial z$ significantly differ. This 343 finding agrees with previous numerical models ${ }^{26,43}$ and intrin344 sically points out that both $|\mathbf{B}|$ and $\nabla \mathbf{B}$ have an impact on the 345 plasma behavior

\section{B. LP measurements}

Plasma properties are characterized using the diagnos348 tics described in Section III. The region delimited by ${ }_{349} 0<y<4 \mathrm{~cm}$ and $-4<z<21 \mathrm{~cm}$ is probed by means of the 350 rf-compensated LP. I-V characteristics are recorded with a 351 spatial resolution of $5 \mathrm{~mm}$. Data is post-processed as dis352 cussed in Section III A and eventually interpolated to enhance 353 visualization. Figures 9, 10 and 11 illustrate normalized 2D 354 maps of $n_{p}, T_{e}$ and $V_{p}$, respectively, for the five magnetic 355 topologies. Normalization values are: $6 \times 10^{17} \mathrm{~m}^{-3}$ as re356 gards to $n_{p}, 6 \mathrm{eV}$ for $T_{e}$ and $25 \mathrm{~V}$ for $V_{p}$. The solid black lines 


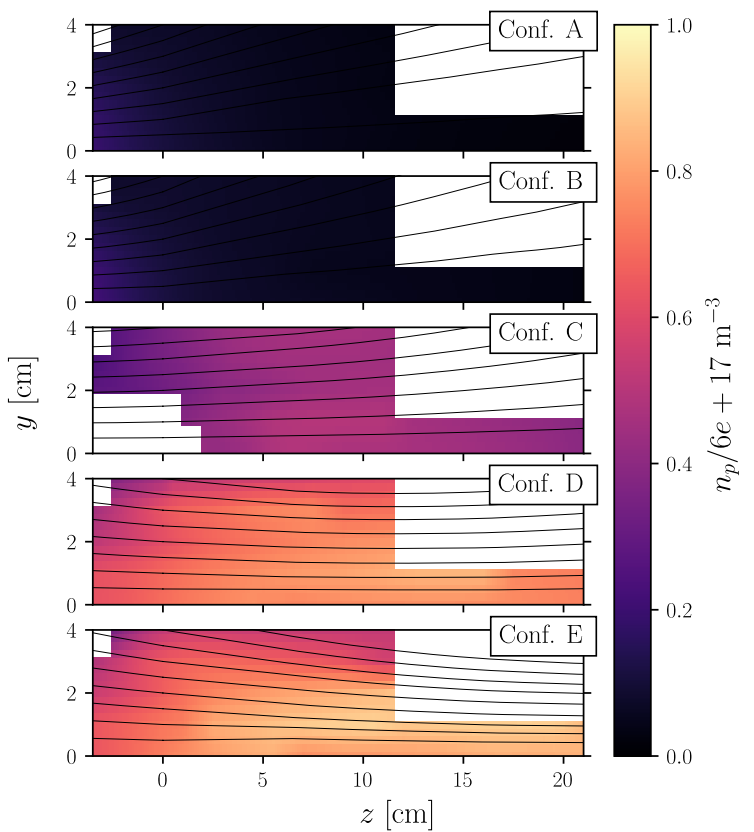

FIG. 9. Normalized plasma density for the five magnetic configurations. Normalization factor is $6 \times 10^{17} \mathrm{~m}^{-3}$. Solid black lines represent magnetic streamlines.

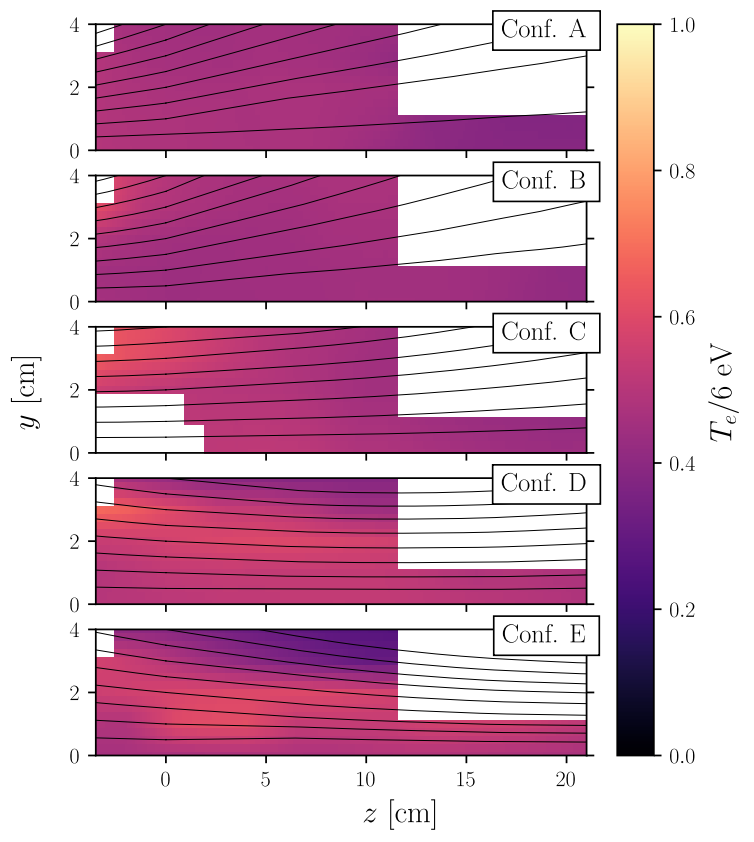

FIG. 10. Normalized electron temperature for the five magnetic con- 379 figurations. Normalization factor is $6 \mathrm{eV}$. Solid black lines represent 380 magnetic streamlines.

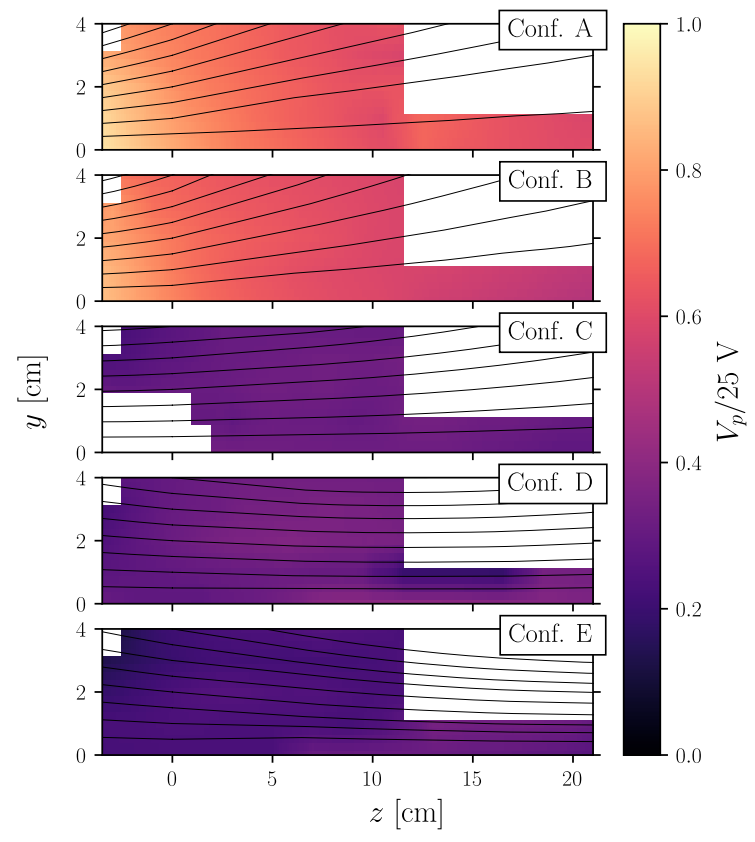

FIG. 11. Normalized plasma potential for the five magnetic configurations. Normalization factor is $25 \mathrm{~V}$. Solid black lines represent magnetic streamlines.

357 included in the figures represent the magnetic streamlines de358 limited by the discharge tube edge at $z=0$. Furthermore, on359 axis profiles of $n_{p}, T_{e}$ and $V_{p}$ are shown in Figure 12. These 360 experimental measurements show that:

1. when the HP source operates in Conf. A, the extracted plasma plume is characterized by a low monotonically decreasing profile of $n_{p}$ ranging between $\sim 1 \times 10^{17}$ and $\sim 9 \times 10^{15} \mathrm{~m}^{-3}$ along the reactor axis. The profile of $T_{e}$ features a monotonic behavior as well, with a peak value of $\sim 3 \mathrm{eV}$. In a similar fashion, $V_{p}$ drops from $\sim 24 \mathrm{~V}$ to $\sim 15 \mathrm{~V}$. All plasma properties peak onaxis at $z<0$. These quantitative observations agree with the faint light emitted in the nozzle region, as stated beforehand. Visual check of the plasma also reveals that light is instead concentrated within the discharge tube. It suggests that the radial and back boundaries represent a major loss term of plasma ${ }^{26,44}$.

2. when the HP source operates in Conf. B, analogous arguments can be put forward. The profile of $n_{p}$ likewise peaks on-axis at $z<0$ and decays monotonically downstream. Altogether, slightly larger values of $n_{p}$ are measured with respect to Conf. A. In the bulk of the plasma, $T_{e}$ remains in the order of $\sim 3 \mathrm{eV}$ with lower values downstream. However, a population of relatively more energetic electrons $\left(T_{e} \sim 3.5 \mathrm{eV}\right)$ appears off-axis, in correspondence of the magnetic nozzle edge, visible at $z<0$ in Figure 10(b). Although qualitatively very 
similar, the distribution of $V_{p}$ for Conf. B presents lower values with respect to Conf. A of a few volts.

3. when the HP source operates in Conf. C, results similar to those previously reported in ${ }^{11}$ using the same HP source are obtained. As a matter of fact, in both the experiments the magnetic throat is located at $z=0$. In contrast to what was discussed in points (i) and (ii), all plasma properties peak off-axis. Data acquisition in the region $(0<r<2, z<0)$ was not possible due to discharge instability possibly induced by the probe presence. The largest value of $n_{p}$ is recorded at $z>0$ and it reads $\sim 3 \times 10^{17} \mathrm{~m}^{-3}$, whereas $T_{e}$ reaches a peak of $4 \mathrm{eV}$ at $z<0$. The distribution of $V_{p}$ is relatively isotropic, ranging in the $8 \pm 0.5 \mathrm{~V}$ interval. The reason why $n_{p}$ and $T_{e}$ present separated regions of the respective maximum values has been explained in ${ }^{11,45}$ in terms of pressure balance. When considering the $z$ component of the electron momentum conservation equation in the limit of negligible contribution due to momentum transfer collisions and due to the Lorenz force, the potential gradient and the pressure gradient balance each other. In the region where $B_{r} / B_{z} \ll 1$, i.e. where the local magnetic field divergence angle is small, the reduced cross field electron mobility yields a relatively large axial conductivity, resulting in a nearly null potential gradient. Hence, for equilibrium reasons, the pressure gradient is required to be small, which results in a larger $n_{p}$ where $T_{e}$ is lower and vice versa. Similar results are numerically found and discussed $\mathrm{in}^{46}$.

4. when the HP source operates in Conf. D, light emission upstream the antenna is almost absent, hinting that plasma losses at the back and lateral walls are mitigated with respect to the previous cases. With reference to Figure $9(\mathrm{~d})$, it is evident that $n_{p}$ follows the axial gradient of $B_{z}$. The maximum value of $\sim 5 \times 10^{17} \mathrm{~m}^{-3}$ is therefore located in correspondence of the magnetic throat. In Figure 10(d), it is relevant to notice the distinct transport pattern of a relatively more energetic electron population along the magnetic field lines at the edge of the nozzle, which exhibits $T_{e} \approx 3.5 \div 4.2 \mathrm{eV}$. A relatively small $V_{p}$ is measured throughout the probed region, with a peak value in the order of $10 \mathrm{~V}$ in the area of maximum $n_{p}$.

5. when the HP source operates in Conf. E, similar observations to those stated in point (4) can be raised. Profiles of $n_{p}$ and $T_{e}$ have a strong dependence on the spatial distribution of $B_{r}$ and $B_{z}$. The highest plasma density value among all the studied magnetic configurations is herein recorded to be $\sim 5.5 \times 10^{17} \mathrm{~m}^{-3}$ nearby the magnetic throat. As highlighted in point (4), also in this case a more energetic population of electrons is transported along the external surface of the magnetic ${ }^{44}$ nozzle, cf. Figure $10(\mathrm{e})$. At $z \cong 10 \mathrm{~cm}$, all the electrons with $T_{e} \gtrsim 3 \mathrm{eV}$ are concentrated in a radius of about $3 \mathrm{~cm}$, as visually confirmed by Figure $8(\mathrm{e})$.

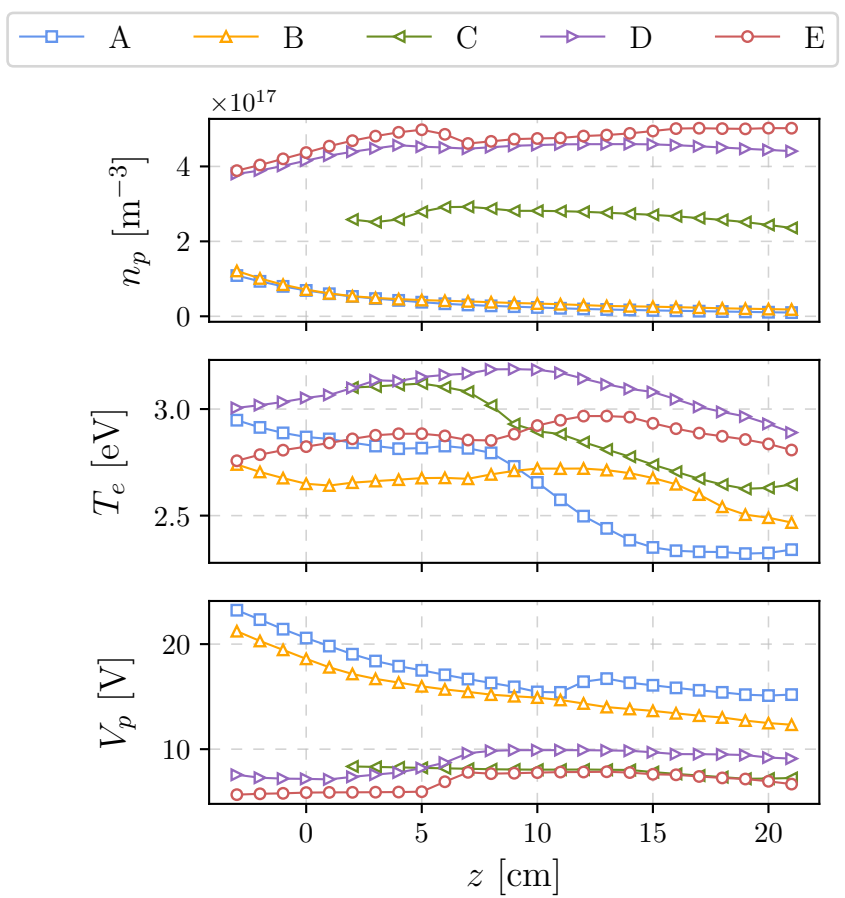

FIG. 12. On-axis profiles of (top) plasma density, (middle) electron temperature and (bottom) plasma potential. Data is extracted from Figures 9-11 and down-sampled to enhance visualization.

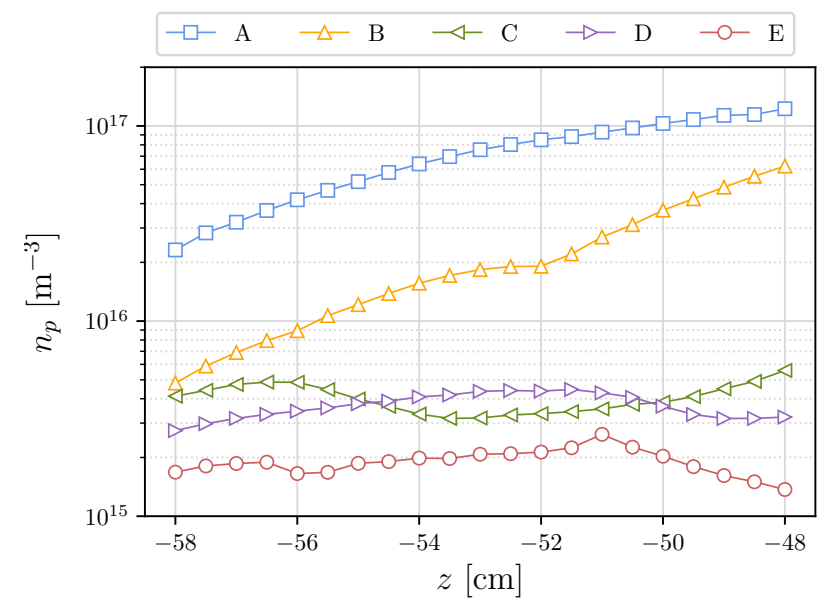

FIG. 13. Ion density profile in the proximity of the back-plate for the five magnetic configurations.

Eventually, it is worth observing that the different config440 urations of $B$ possibly influence the power deposition map. ${ }_{441}$ This, in turn, very likely relates to the different spatial distri42 butions of $n_{p}$ and $T_{e}$ as discussed in points (1)-(5).

In addition to the measurements performed using the rf444 compensated LP, a simple uncompensated LP is inserted on445 axis at the back/injection plate of the HP source to measure ${ }_{446} n_{p}$ as an indicator of the plasma flux lost in this region. The ${ }_{447}$ probe is biased to scan over the ion saturation branch only. 
${ }_{448}$ No significant distortions due to rf oscillations are expected in ${ }_{449}$ this part of the I-V curve ${ }^{33}$. For obtaining this particular set ${ }_{450}$ of data, the reactor injection plate has been modified in order 451 to accommodate the probe and its related wiring. The pro${ }_{452}$ pellant is still injected off-axis but it goes through a plenum ${ }_{453}$ first and eventually reaches the discharge tube via a multi${ }_{454}$ perforated surface, resulting in a axial gas flow. An uncom455 pensated probe is chosen due to limitations on the available ${ }_{456}$ room. The measured profiles of $n_{p}$ are shown in Figure 13 ${ }_{457}$ for Conf. A to E. Since the magnetic throat in Conf. A is lo${ }_{458}$ cated upstream the antenna, the ions are mostly transported 459 towards the back plate by following the convergent-divergent 460 shape of the magnetic field. As the magnetic throat moves far461 ther downstream from the antenna center, lower densities are ${ }_{462}$ measured in the proximity of the injection plate, as expected.

\section{${ }_{463}$ C. Plasma density profile model}

${ }_{464}$ Using a simplified description of the plasma, the measured 465 axial profiles of $n_{p}$ can be compared with theoretical profiles ${ }_{466}$ retrieved from the helicon wave dispersion relation, which is ${ }_{467}$ thereafter derived accounting for the non-uniformity of the ex468 ternal magnetic field along $z$. This approach implicitly as${ }_{469}$ sumes that the plasma production entirely relies on the wave470 mode power coupling. Although the helicon regime is not 471 proven here by direct measurement of the excited wave, previ${ }_{472}$ ous experiments performed on the same device operated with 473 similar ranges of magnetic field strength and input power have ${ }_{474}$ shown the presence of the propagating wave ${ }^{11}$. Considering 475 that the electric $\tilde{\mathbf{E}}$ and magnetic $\tilde{\mathbf{B}}$ components of the exited 476 wave are described ${ }^{47,48}$ as

$$
\tilde{\mathbf{E}}, \tilde{\mathbf{B}} \sim \exp \left[i\left(m \theta+k_{z} z-\omega t\right)\right],
$$

477 where $m$ is the azimuthal mode number, $\theta$ is the azimuthal 478 angle and $k_{z}$ is the axial wavenumber, Maxwell equations as479 sume the form

$$
\nabla \cdot \tilde{\mathbf{B}}=0
$$

$$
\nabla \times \tilde{\mathbf{E}}=i \omega \tilde{\mathbf{B}}
$$

481

$$
\nabla \times \tilde{\mathbf{B}}=\mu_{0} \mathbf{j}
$$

482

The electron momentum equation is written as

$$
-e n_{p} \tilde{\mathbf{E}}=i \frac{m_{e}}{e} \mathbf{j}_{e}(\omega+i v)-\mathbf{j}_{e} \times \mathbf{B}
$$

${ }_{483}$ where $\mathbf{j}_{e}$ is the electron current density, $\mathbf{B}$ is the external mag- ${ }^{516}$ ${ }_{484}$ netic field and $v$ is a collision rate accounting for all dissi- ${ }^{517}$ 485 pation phenomena. Additional comments and derivation of 518 ${ }_{486}$ Equation 3 are reported $\mathrm{in}^{47}$. The widespread ideal assump- ${ }_{519}$ ${ }_{487}$ tion of perfectly uniform magnetic field is not appropriate in 520 488 the actual case. Therefore, although still simplistic, it is as- ${ }_{521}$ 489 sumed that $\mathbf{B}=B(z) \hat{\mathbf{z}}$, i.e. only the variation of the magnetic ${ }_{522}$
TABLE II. Helicon wave parameters at $z=10 \mathrm{~cm}$.

\begin{tabular}{llllll}
\hline \hline & Conf. A & Conf. B & Conf. C & Conf. D & Conf. E \\
\hline$r_{p}[\mathrm{~cm}]$ & 7.3 & 8.1 & 5.1 & 4.0 & 3.5 \\
$k_{z}\left[\mathrm{~cm}^{-1}\right]$ & 0.26 & 0.26 & 0.26 & 0.26 & 0.26 \\
$\lambda_{z}[\mathrm{~cm}]$ & 24 & 24 & 24 & 24 & 24 \\
$\kappa_{1}\left[\mathrm{~cm}^{-1}\right]$ & 0.46 & 0.41 & 0.68 & 0.88 & 1.03 \\
$\kappa_{2}\left[\mathrm{~cm}^{-1}\right]$ & 0.14 & 0.17 & 0.07 & -0.01 & -0.08 \\
\hline \hline
\end{tabular}

490 field along $z$ is accounted for. Combining Equation 2a-(2c) 491 and Equation 3, one obtains

$$
\begin{aligned}
&\left(\frac{\omega+i v}{\omega_{c_{e}}}\right) \nabla \times(\nabla \times \tilde{\mathbf{B}})+k_{z} \nabla \times \tilde{\mathbf{B}}+ \\
&+\frac{\mu_{0} \omega e n_{p}}{B} \tilde{\mathbf{B}}=\mathbf{0},
\end{aligned}
$$

492 where the explicit dependence on $z$ has been omitted, $\omega_{c_{e}}$ is 493 the electron cyclotron frequency and the rest is conventional. 494 Detailed derivation of Equation 4 is likewise included in $^{47}$. 495 Eventually, in the limit of $m_{e} \rightarrow 0$, the local dispersion relation 496 is retrieved in the same shape of the well known OD dispersion 497 relation, i.e.

$$
k(z) k_{z}=\frac{\mu_{0} \operatorname{\omega en}_{p}(z)}{B(z)},
$$

498 where $k(z)$ indicates the total wavenumber. The wave bound99 ary conditions are fixed by ${ }^{48}$

$$
m k(z) \mathbf{J}_{m}\left[k_{r}(z) r_{p}(z)\right]+k_{z} \mathbf{J}_{m}^{\prime}\left[k_{r}(z) r_{p}(z)\right]=0,
$$

500 where $\mathbf{J}_{m}$ and $\mathbf{J}_{m}^{\prime}$ are the Bessel function of the first kind 501 of order $m$ and its derivative, respectively, $k_{r}(z)$ is the lo502 cal radial wavenumber and $r_{p}(z)$ is the local plasma ra503 dius. The value of $k_{z}$ is fixed by the antenna length, 504 i.e. $k_{z}=\pi / d_{A}, 3 \pi / d_{A}, 5 \pi / d_{A}$, etc. Pretending that only the 505 first order axial mode is coupled with the antenna, the compu506 tation algorithm includes:

- the axial wavenumber is estimated as $k_{z}=\pi / d_{A}$, therefore the axial wavelenth is $\lambda_{z}=24 \mathrm{~cm}$;

- $r_{p}(z)$ is set equal to the radius of the magnetic streamline crossing the tube edge at $z=0$, see Figure 2;

- considering the geometry of the antenna and the direction of the external magnetic field, it is imposed that $m=+1$;

- two cases are distinguished:

1. $k_{r}(z)$ is computed numerically from Equation 6 and it is named as $\kappa_{1}(z)$ for the sake of clarity in the following;

2. $k_{r}(z)$ is evaluated as $\kappa_{1}(z) \sin [\alpha(z)]$ referred to as $\kappa_{2}(z)$ hereafter. Here $\alpha(z)$ is the local magnetic field divergence angle. The purpose of this heuristic strategy is to provide the model with the information that the applied magnetic field is not 


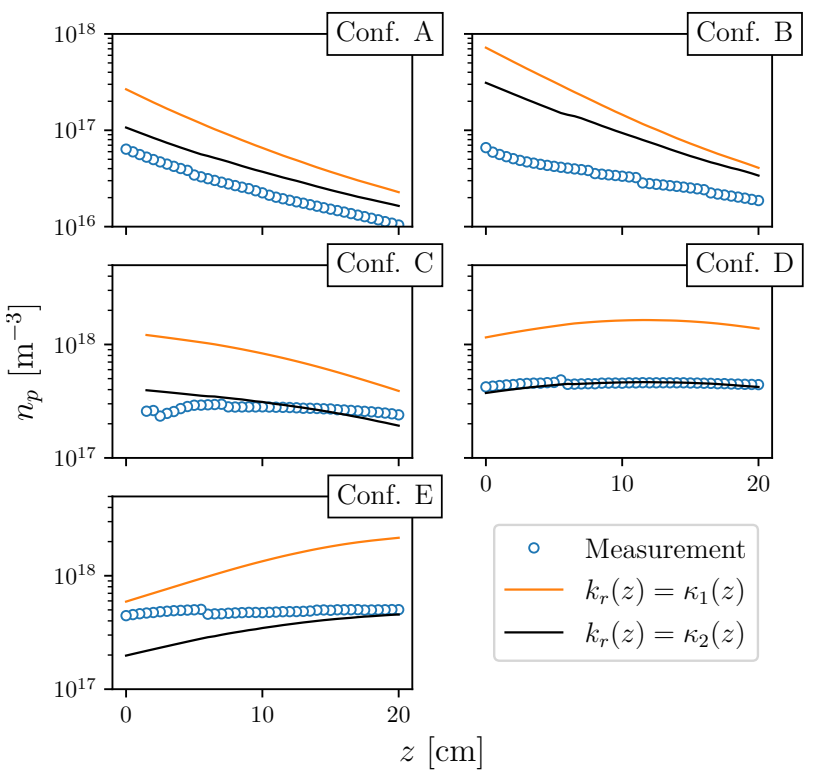

FIG. 14. Plasma density at $r=0$ computed using the helicon waves dispersion relation in the $k_{r}(z)=\kappa_{1}(z)$ and $k_{r}(z)=\kappa_{2}(z)$ cases compared to measurement data.

purely axial. In doing so, it is postulated that the helicon wave resonance cone reduces to a line at the magnetic throat, i.e. where $\alpha=0$, thus $k$ equals $k_{z}$.

- the profile $n_{p}(z)$ is consequently computed from Equation 5 for the aforementioned two cases.

529 Numerical values of the relevant parameters are reported 530 in Table II for all magnetic configurations. In Figure 14, the 531 obtained theoretical curves are compared with measurements ${ }_{532}$ of $n_{p}$ extracted from Figure 9 at $x, y=0$ for all the tested ${ }_{553}$ 533 magnetic configurations. In general, it is found that the case ${ }_{554}$ ${ }_{534} k_{r}(z)=\kappa_{1}(z)$ provides an overestimation of the plasma den- 555 535 sity. When dealing with Conf.s D and E, the $k_{r}(z)=\kappa_{2}(z)$ case ${ }_{556}$ tion III. The probe is displaced radially along the $x$ axis 536 is in very good agreement with the data in correspondence 557 at $z=5 \mathrm{~cm}$ and $z=15 \mathrm{~cm}$ in order to analyze the diver${ }_{537}$ of the magnetic throat, that is where the strong assumption 558 gence of the extracted plasma plume. The measurements of ${ }_{538} \mathbf{B}=B(z) \hat{\mathbf{z}}$ is more representative. Ultimately, $n_{p}$ measured in $559 j_{i}$ are shown in Figure 15(a-b). Table III gathers the val${ }_{539}$ Conf. $\mathrm{C}$ is in relatively good agreement with the latter case. 560 ues of the divergence parameter $\delta$ computed as the ratio ${ }_{540}$ It is noted that even the $k_{r}(z)=\kappa_{2}(z)$ case overestimates the $561 j_{i}(x=-11.5 \mathrm{~cm}) / j_{i}(x=0 \mathrm{~cm})$. Results confirm that a low${ }_{541}$ measured values of $n_{p}$ for Conf.s A and B, although match- ${ }_{562}$ current high-divergence ion beam is extracted from the HP 542 ing the qualitative profile. This suggests that the helicon wave ${ }_{563}$ source when operating in Conf. A and B. Differently, the ion ${ }_{543}$ is evanescent in this region of the plasma since the measured 564 beams related to Conf.s C, D and E feature a similar low${ }_{544} n_{p}$ is lower than the one required by the dispersion relation. 565 divergence profile of $j_{i}$ in the proximity of the tube exit, see 545 In conclusion, these profiles of $n_{p}$ computed from the disper- 566 Figure 15(a). Yet, the divergence angle of Conf. C rapidly ${ }_{546}$ sion relation represent a first effective estimation of the actual ${ }_{567}$ grows as the plasma expands downstream, cf. Figure 15(b). ${ }_{547}$ spatial evolution of plasma density. Therefore, these quanti- 568 The highest value of $j_{i}$ is recorded in Conf.E at $z=15 \mathrm{~cm}$, ${ }_{548}$ tative information can be of some convenience for guessing 569 in agreement with the largest $n_{p}$ probed in the same configu${ }_{549}$ the propagation region of helicon waves and for preliminary 570 ration. Larger values of $j_{i}$ relate well with higher propellant 550 modeling of the plasma dynamics and/or design of the axial 571 utilization efficiency, which is a desirable working condition 551 magnetic field profile.
572 for space propulsion applications. 
TABLE IV. Order of magnitude of relevant velocity quantities.

\begin{tabular}{ll}
\hline \hline & $\mathscr{O}(v)$ \\
\hline Ion acoustic velocity & $v_{B}=\sqrt{k T_{e} / m_{i}} \approx 2000 \mathrm{~m} \mathrm{~s}^{-1}$ \\
Thermal velocity & $v_{t h}=\sqrt{8 k T_{i} / \pi m_{i}} \approx 275 \mathrm{~m} \mathrm{~s}^{-1}$ \\
\hline \hline
\end{tabular}

\section{${ }_{573}$ E. LIF measurements}

574 Using LIF spectroscopy, the velocity distribution functions 575 of krypton atoms $\mathrm{Kr}$ I and ions $\mathrm{Kr}$ II are inferred. Infor576 mation about the velocity of the ions is of particular inter577 est for driving conclusions about propulsive performance. On 578 the other hand, knowledge of the atoms velocity is useful for 579 modeling of the plasma flow. Table IV exemplifies the or${ }_{580}$ der of magnitude of the relevant velocity terms in the HP ${ }_{581}$ source operating with $\mathrm{Kr}$. Radial profiles of $\mathrm{Kr}$ I VDF are 582 evaluated at $z=12 \mathrm{~cm}$ and shown in Figure 16 for all mag${ }_{583}$ netic configurations. The spatial resolution is $10 \mathrm{~mm}$. In this ${ }_{584}$ case, the reported data did not require any post-processing as 585 a adequate signal-to-noise ratio was found. These measure586 ments show that the most probable velocity of $\mathrm{Kr} \mathrm{I}$ is nearly 587 unchanged over the different magnetic topologies, reading ${ }_{588} \sim 250 \div 300 \mathrm{~m} \mathrm{~s}^{-1}$, therefore in the order of the thermal ve${ }_{589}$ locity assuming $T_{i} \approx 300 \mathrm{~K}$, cf. Table IV. No dependence of 590 the velocity on the radial location $y$ is recorded. Dissimilar 591 values of velocity spread are found between the magnetic con592 figurations, with a broader VDF for Conf. C and Conf. D.

${ }_{593}$ Axial VDFs of $\mathrm{Kr}$ II are probed in the same radial region ${ }_{594}$ with a spatial resolution of $5 \mathrm{~mm}$ at three distinct axial posi595 tions, i.e. $z=10.5 \mathrm{~cm}, z=12 \mathrm{~cm}$ and $z=25 \mathrm{~cm}$. At some $596 y$ locations, the LIF signal was not distinguishable from the 597 background noise due to small local values of $n_{p}$, therefore ${ }_{598}$ mapping of the VDFs was not possible. For the very same 599 reason, Conf.s A and B did not allow obtaining an appropri600 ate signal-to-noise ratio in any of the probed locations. Raw ${ }_{601}$ VDFs are post-processed using a least squares Gaussian fit. 602 The resulting profiles when the HP source operates in Conf.s ${ }_{603} \mathrm{C}, \mathrm{D}$ and $\mathrm{E}$ are reported in Figure 17, 18 and 19, respectively. ${ }_{604}$ In addition, the most probable velocity is extracted and plot 605 in Figure 20 thus to facilitate comparison among the various 606 magnetic configurations. It is noticed that the profiles related 607 to Conf.s C and D feature a nearly identical trend, whereas the 608 ion velocity in Conf. E generally retains lower readings. This 609 is in agreement with the measured values of $V_{p}$ which remain ${ }_{610}$ below those concerning Conf. $\mathrm{C}$ and $\mathrm{D}$, cf. Figure 11. In the 611 further upstream regions, namely $z=10.5 \mathrm{~cm}$ and $z=12 \mathrm{~cm}$, ${ }_{612}$ velocities smaller than $\sim 250 \mathrm{~m} \mathrm{~s}^{-1}$ are measured, therefore in ${ }_{613}$ the order of the thermal velocity assuming $T_{i} \approx 300 \mathrm{~K}$. Con614 versely, at $z=25 \mathrm{~cm}$, all the velocities increase by a factor of ${ }_{622}$ size of the vacuum chamber. Similar small values of ion ve${ }_{615} 2$ to 3 , reaching values as high as $\sim 500 \mathrm{~m} \mathrm{~s}^{-1}$. These small ${ }_{623}$ locity are reported in Ref. 49 using LIF on a large scale HP ${ }_{616}$ velocities somewhat concur with the fact that the magnetic ${ }_{624}$ source. In all of the magnetic configurations, the drop of $V_{p}$ ${ }_{617}$ field is weakly divergent in the probed region for Conf.s $\mathrm{C}$ and ${ }_{625}$ is fairly small (in the order of $1 \mathrm{~V}$ ) within $z<21 \mathrm{~cm}$, cf. Fig${ }_{618} \mathrm{D}$, while $z=25 \mathrm{~cm}$ represents the throat location for Conf. E. ${ }_{626}$ ure 11. Considering Conf. E for instance, since at $z<25 \mathrm{~cm}$ ${ }_{619}$ A weakly divergent magnetic field does not allow for a signifi- ${ }_{227}$ the magnetic field is convergent, it is expected that the favored ${ }_{620}$ cant momentum gain imparted by the Lorentz force. Measure- 628 inward transport of electrons do not allow for a significant po${ }_{621}$ ments farther downstream were prevented due to the limited 629 tential drop in this region. In the purely electrostatic case, 


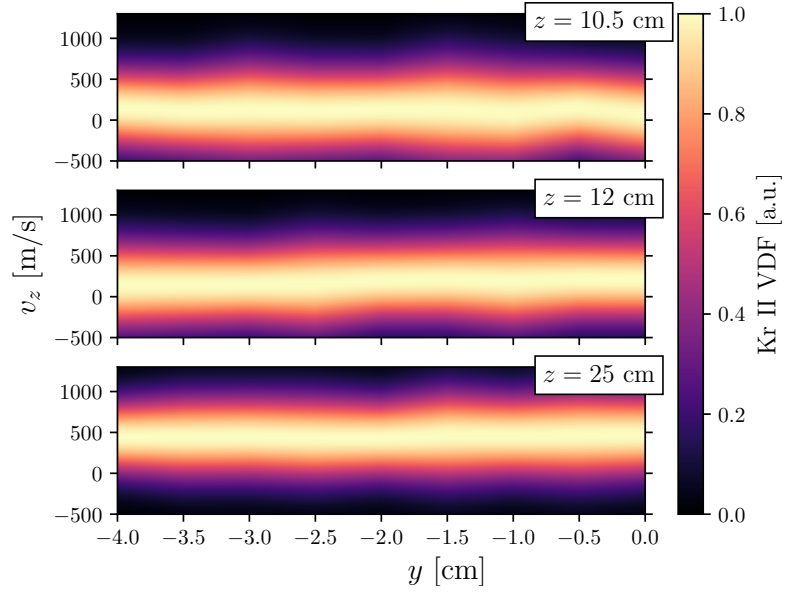

FIG. 18. Kr II velocity distribution function for Conf. D measured along the $y$ axis at $z=10.5,12$ and $25 \mathrm{~cm}$.

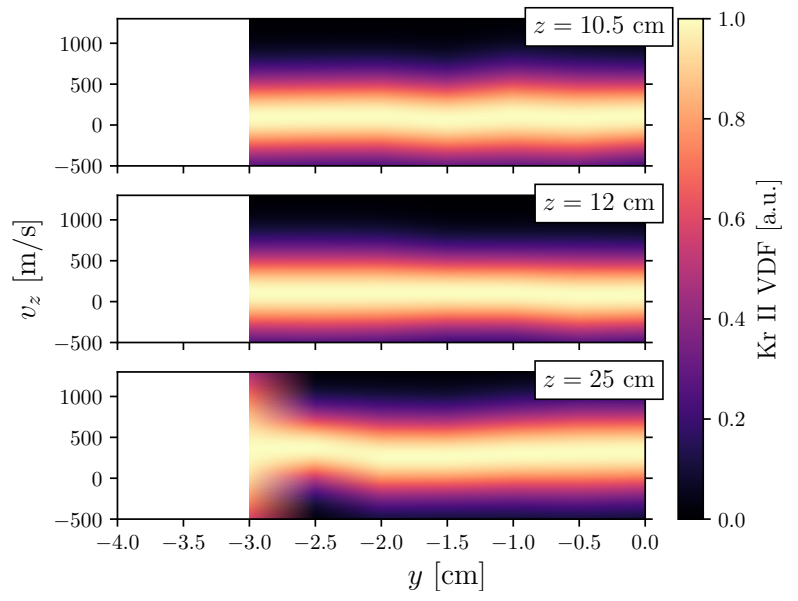

FIG. 19. Kr II velocity distribution function for Conf. E measured along the $y$ axis at $z=10.5,12$ and $25 \mathrm{~cm}$.

630 a potential drop of $\sim 1 \mathrm{~V}$ translates into an ion velocity in${ }_{631}$ crease of $\sim 1.5 \mathrm{~km} \mathrm{~s}^{-1}$. Hence, the acceleration of the ions 662 the mechanisms occurring within the nozzle. The exploited ${ }_{632}$ throughout the $\mathrm{MN}$ does not fully exploit the available en- ${ }_{663}$ parameters are: input power $(750 \mathrm{~W}$ and $900 \mathrm{~W})$; mass flow ${ }_{633}$ ergy. This phenomenon is related to some dissipation phe- 664 rate $\left(0.5 \mathrm{mg} \mathrm{s}^{-1}, 1 \mathrm{mg} \mathrm{s}^{-1}\right.$ and $\left.2 \mathrm{mg} \mathrm{s}^{-1}\right)$; current in the elec${ }_{634}$ nomenon, e.g. collisions, as later discussed. Moreover, al- 665 tromagnets ( $6 \mathrm{~A}$ and $8 \mathrm{~A}$ ). The magnetic configuration is fixed 635 though no sonic condition is achieved at the magnetic throat 666 to Conf. D for this experiment because of its divergent shape ${ }_{636}$ in Conf.s $\mathrm{C}$ and $\mathrm{D}$, ions experience a further acceleration in 667 at $z=25 \mathrm{~cm}$ and larger signal-to-noise ratio with respect to ${ }_{637}$ the divergent part of the nozzle, see Figure 20. It suggests that ${ }_{668}$ Conf. C. The resulting VDFs are shown in Figure 21, which ${ }_{638}$ the sonic point, if ever reached, is shifted downstream, in con- 669 includes the raw data, the Gaussian fittings and the unfold 639 trast with the ideal postulation of gas-dynamics-like plasma 670 most probable velocities obtained at each working condition. ${ }_{640}$ expansion $^{50}$. In this experiment, the ions might reach the ${ }_{671}$ The overall tendency results clear. Indeed, higher ion veloci${ }_{641}$ sonic speed only at the sheath edge that forms downstream 672 ties are attained as the mass flow rate decreases and the mag642 between the chamber wall and the plasma plume. Numer- 673 netic field is increased, whereas slower ions are recorded with ${ }_{643}$ ous works focusing on modeling of magnetic nozzle dynam- 674 larger mass flow rates. These outcomes suggest that collisions ${ }_{644}$ ics have assumed that the ion Mach number equals unity at 675 resulting from a larger pressure do play a role in the acceler${ }_{645}$ the throat ${ }^{51-53}$. Albeit this condition conveniently allows sep- 676 ation process. At lower pressures, a larger mean free path is ${ }_{646}$ arating the processes of plasma generation and plasma accel- ${ }_{677}$ expected, therefore allowing for greater values of $T_{e}$. 


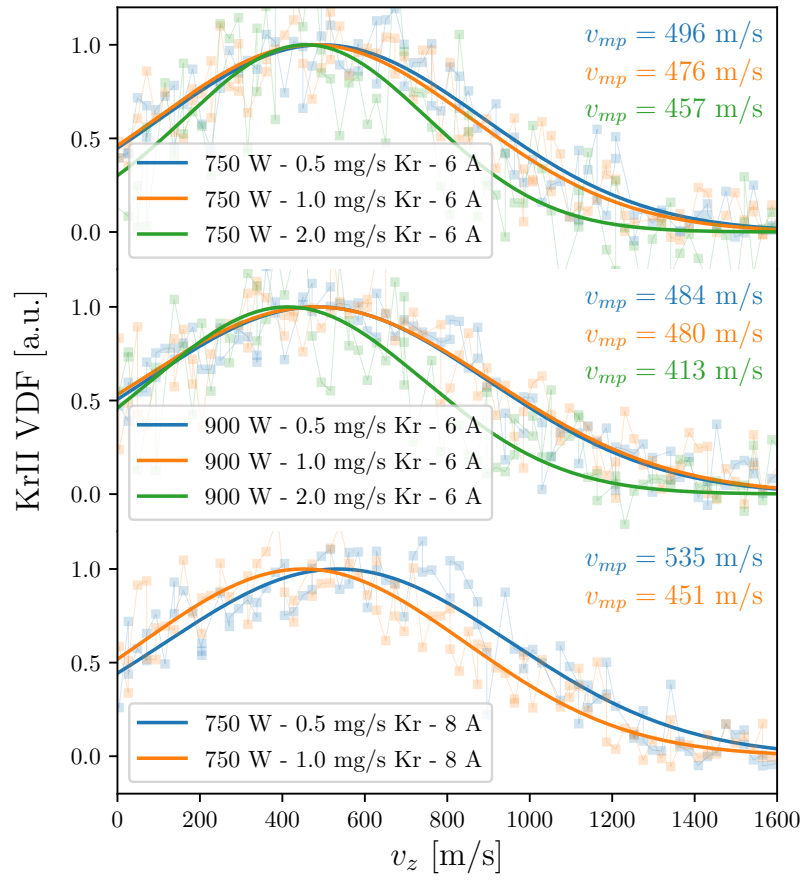

703 the helicon wave propagation region. Furthermore, prelim704 inary modeling and design of Helicon thrusters can perhaps 705 make use of this approach to shape the axial magnetic field 706 profile.

${ }_{707}$ The measured ion axial velocities exhibit small values, be708 ing below the sonic condition at the magnetic throat. By mea709 suring the most probable ion velocity obtained under differ710 ent working parameters (input power, magnetic strength and 711 mass flow rate), it is concluded that ionization and collisions 712 occurring in the near-field plume contribute to limiting the ion 713 acceleration process.

\section{${ }_{714}$ ACKNOWLEDGMENTS}

715 This project has received funding from the European 716 Union's Horizon 2020 research and innovation program un717 der grant agreement No 870542 (HelIcon PlasmA Thruster 718 for In-space Applications).

\section{${ }_{719}$ CONFLICT OF INTEREST}

720 The authors have no conflicts to disclose.

\section{DATA AVAILABILITY}

FIG. 21. Kr II axial VDF for Conf. D at $z=25 \mathrm{~cm}$ as function of
$P_{I N}, \dot{m}$ and electromagnets current. Scatter points indicate raw data, solid lines exemplify least squares Gaussian fittings.

\section{V. CONCLUSION}

679 In summary, several plasma properties are inferred via di680 rect measurement to establish empirical approaches to thruster 681 design and optimization. The magnetic throat is displaced 682 along the reactor axis and the resultant plasma is studied in ${ }_{683}$ the near-field plume using electrostatic diagnostics and LIF ${ }_{684}$ spectroscopy. It is found that a low-current high-divergence 685 plume is extracted from the plasma source when the magnetic 686 throat is located upstream or in correspondence of the antenna 687 center point. Plasma density measurements at the back plate 688 emphasize that, in those cases, the generated plasma mainly 689 remains within the discharge tube and is lost at the radial and ${ }_{690}$ back boundaries. Larger ion currents and higher confinement ${ }_{691}$ levels are attained when the magnetic throat is located down692 stream the antenna, namely at a distance at least equal to the 693 wavelength corresponding to the first order axial mode of the 694 helicon wave. Hence, optimization of the plasma generation ${ }_{695}$ process in a Helicon thruster would include the separation of ${ }_{696}$ the magnetic throat from the antenna location in accordance 697 with the excited helicon wavelength.

698 Theoretical axial profiles for the plasma density are directly 699 derived from the dispersion relation under the hypothesis of 700 non-uniform magnetic field. Comparison with probe mea701 surements show relatively good agreement therefore hinting 702 the applicability of this theoretical description for guessing
The data that support the findings of this study are available ${ }_{23}$ from the corresponding author upon reasonable request.

\section{REFERENCES}

${ }^{1}$ Igor Levchenko, Kateryna Bazaka, Yongjie Ding, Yevgeny Raitses, Stéphane Mazouffre, Torsten Henning, Peter J. Klar, Shunjiro Shinohara, Jochen Schein, Laurent Garrigues, Minkwan Kim, Dan Lev, Francesco Taccogna, Rod W. Boswell, Christine Charles, Hiroyuki Koizumi, Yan Shen, Carsten Scharlemann, Michael Keidar, and Shuyan Xu. Space micropropulsion systems for Cubesats and small satellites: From proximate targets to furthermost frontiers. Applied Physics Reviews, 5(1), 2018.

2Javier Martínez Martínez, Dmytro Rafalskyi, and Ane Aanesland. Development and Testing of the NPT30-I2 Iodine Ion Thruster. In 36th International Electric Propulsion Conference, 2019.

${ }^{3}$ Antonio Gurciullo, Julien Jarrige, Paul Lascombes, and Denis Packan. Experimental performance and plume characterisation of a miniaturised $50 \mathrm{~W}$ Hall thruster. In 36th International Electric Propulsion Conference, 2019.

${ }^{4}$ I. Levchenko, S. Xu, S. Mazouffre, D. Lev, D. Pedrini, D. Goebel, L. Garrigues, F. Taccogna, and K. Bazaka. Perspectives, frontiers, and new horizons for plasma-based space electric propulsion. Physics of Plasmas, 27(2), 2020

${ }^{5}$ E. Dale, B. Jorns, and A. Gallimore. Future Directions for Electric Propul-

${ }^{6}$ David Krejci, Lou Grimaud, Tony Schönherr, Valentin Hugonnaud, Alexander Reissner, and Bernhard Seifert. ENPULSION NANO and MICRO propulsion systems: development and testing. In AIAA Propulsion and Energy Forum, 2021

${ }^{7}$ Dillon O'reilly, Georg Herdrich, and Darren F. Kavanagh. Electric propul-

${ }^{8}$ S. N. Bathgate, M. M.M. Bilek, and D. R. McKenzie. Electrodeless plasma thrusters for spacecraft: A review. Plasma Science and Technology, 19(8), 2017 sion Research. Aerospace, 7(120), 2020. sion methods for small satellites: A review. Aerospace, 8(1):1-30, 2021. 
$753{ }^{9}$ Shunjiro Shinohara. Helicon high-density plasma sources: Physics and 820 applications. Advances in Physics: X, 3(1):185-220, 2018.

$755{ }^{10} \mathrm{~K}$. Takahashi. Helicon-type radiofrequency plasma thrusters and magnetic 756 plasma nozzles. In Reviews of Modern Plasma Physics, volume 3. Springer 57 Singapore, 2019.

${ }_{758}{ }^{11}$ Alfio E Vinci and Stéphane Mazouffre. Direct experimental comparison of

59 krypton and xenon discharge properties in the magnetic nozzle of a helicon

plasma source. Physics of Plasmas, 28(033504), 2021.

${ }_{761}{ }^{12}$ Kazunori Takahashi. Magnetic nozzle radiofrequency plasma thruster approaching twenty percent thruster efficiency. Scientific Reports, 11(1):1-12, 2021.

$764{ }^{13}$ Nicolas Bellomo, Mirko Magarotto, Marco Manente, Fabio Trezzolani,

765 Riccardo Mantellato, Lorenzo Cappellini, Devis Paulon, Antonio Selmo,

766 Davide Scalzi, Marco Minute, Matteo Duzzi, Alessandro Barbato, Alessan767 dro Schiavon, Simone Di Fede, Nabil Souhair, Paola De Carlo, Francesco

768 Barato, Fabiana Milza, Elena Toson, and Daniele Pavarin. Design and In

769 orbit Demonstration of REGULUS, an Iodine electric propulsion system.

770 CEAS Space Journal, (0123456789), 2021.

${ }_{771}{ }^{14}$ Seth J Thompson, Fort Collins, Casey C Farnell, Shawn C Farnell, Plasma

772 Controls, Fort Collins, Desiree D Williams, John P Chandler, Fort Collins,

773 John D Williams, and Fort Collins. Evaluation of Iodine Compatible Cath-

774 ode Configurations. 36th International Electric Propulsion Conference 775 pages $1-21,2019$.

${ }_{776}{ }^{15}$ M. Light, I. D. Sudit, F. F. Chen, and D. Arnush. Axial propagation of 777 helicon waves. Physics of Plasmas, 2(11):4094-4103, 1995.

$778{ }^{16}$ D. D. Blackwell, T. G. Madziwa, D. Arnush, and F. F. Chen. Evidence for 779 Trivelpiece-Gould Modes in a Helicon Discharge. Physical Review Letters, 88(14):4, 2002

${ }_{781}{ }^{17}$ K. Takahashi, T. Lafleur, C. Charles, P. Alexander, R. W. Boswell, M. Per782 ren, R. Laine, S. Pottinger, V. Lappas, T. Harle, and D. Lamprou. Direct 783 thrust measurement of a permanent magnet helicon double layer thruster. ${ }_{84}$ Applied Physics Letters, 98(14):2-4, 2011.

${ }_{785}{ }^{18} \mathrm{~L}$. T. Williams and M. L.R. Walker. Thrust measurements of a helicon plasma source. 47th AIAA/ASME/SAE/ASEE Joint Propulsion Conference and Exhibit 2011, pages 1-15, 2011.

${ }_{788}{ }^{19} \mathrm{~S}$. Pottinger, V. Lappas, C. Charles, and R. Boswell. Performance characterization of a helicon double layer thruster using direct thrust measurements.

900 Journal of Physics D: Applied Physics, 44(23), 2011.

$791{ }^{20} \mathrm{~K}$. Takahashi, C. Charles, R. Boswell, and A. Ando. Performance improve2 ment of a permanent magnet helicon plasma thruster. Journal of Physics D: Applied Physics, 46(35), 2013.

${ }_{794}{ }^{21}$ Kazunori Takahashi, Yoshinori Takao, and Akira Ando. Thrust imparted by a stepped-diameter magnetic nozzle rf plasma thruster. Applied Physics Letters, 113(3), 2018

79722 J. Navarro-Cavallé, M. Wijnen, P. Fajardo, and E. Ahedo. Experimental characterization of a $1 \mathrm{~kW}$ Helicon Plasma Thruster. Vacuum, 149:69-73, 2018.

$800{ }^{23}$ J. Navarro-Cavallé, M. Wijnen, P. Fajardo, E. Ahedo, V. Gomez 801 A. Giménez, and Mercedes Ruiz. Development and Characterization of 802 the Helicon Plasma Thruster Prototype HPT05M. In 36th International 803 Electric Propulsion Conference, number September, 2019.

${ }_{804}{ }^{24}$ T. A. Collard and B. A. Jorns. Magnetic nozzle efficiency in a low power 805 inductive plasma source. Plasma Sources Science and Technology, 28(10), 805 inductive 2019

${ }_{807}^{25}$ Eduardo Ahedo and Jaume Navarro-Cavallé. Helicon thruster plasma 808 modeling: Two-dimensional fluid-dynamics and propulsive performances. 809 Physics of Plasmas, 20(4), 2013.

${ }_{810}{ }^{26} \mathrm{~T}$. Lafleur. Helicon plasma thruster discharge model. Physics of Plasmas, $811 \quad 21(4), 2014$.

${ }_{812}{ }^{27}$ M. Magarotto, D. Melazzi, and D. Pavarin. Study on the influence of 813 the magnetic field geometry on the power deposition in a helicon plasma 14 source. Journal of Plasma Physics, 85(4):1-19, 2019.

${ }_{815}{ }^{28} \mathrm{~K}$. Takahashi, Y. Takao, and A. Ando. Increased Thrust-to-Power Ratio 816 of a Stepped-Diameter Helicon Plasma Thruster with Krypton Propellant. Journal of Propulsion and Power, 2020.

${ }_{818}{ }^{29}$ J. M. Little and E. Y. Choueiri. Electron Cooling in a Magnetically Expand819 ing Plasma. Physical Review Letters, 117(22):1-5, 2016.
${ }^{30}$ T. Lafleur, C. Charles, and R. W. Boswell. Characterization of a helicon plasma source in low diverging magnetic fields. Journal of Physics D: Applied Physics, 44(5), 2011.

${ }^{31}$ Alexander Bennet, Christine Charles, and Rod Boswell. Non-local plasma generation in a magnetic nozzle. Physics of Plasmas, 26(7), 2019.

${ }^{32}$ I. D. Sudit and F. F. Chen. RF Compensated Probes for High-density Discharges. Plasma Sources Science and Technology, 3(2):162-168, 1994

${ }^{33}$ F. F. Chen. Lectures Notes on Langmuir Probe Diagnostics, 2003.

${ }^{34}$ F. F. Chen. Langmuir probes in RF plasma: Surprising validity of OML theory. Plasma Sources Science and Technology, 18(3), 2009.

${ }^{35}$ F. F. Chen. Langmuir probe analysis for high density plasmas. Physics of Plasmas, 8(6):3029-3041, 2001

${ }^{36}$ S. Mazouffre, G Largeau, L Garrigues, C Boniface, and K Dannenmayer. Evaluation of various probe designs for measuring the ion current density in a Hall thruster plume. 35th International Electric Propulsion Conference, (8-12 October):IEPC-2017-336, 2017.

${ }^{37} \mathrm{~S}$. Mazouffre. Mesure de la densité de courant ionique dans le jet plasma d'un propulseur de Hall. Théorie et instrumentation. Technical report, 2016. ${ }^{38} \mathrm{~S}$. Mazouffre. Laser-induced fluorescence diagnostics of the cross-field discharge of Hall thrusters. Plasma Sources Science and Technology, 22(1), 2012

${ }^{39}$ A. Lejeune, G. Bourgeois, and S. Mazouffre. Kr II and Xe II axial velocity distribution functions in a cross-field ion source. Physics of Plasmas, 19(7), 2012.

${ }^{40}$ A. Kramida, Yu. Ralchenko, J. Reade, and Team NIST ASD NIST Atomic Spectra Database (ver. 5.8), [Online]. Available: https://physics.nist.gov/asd [2021, June 7]. National Institute of Standards and Technology, Gaithersburg, MD, 2020.

${ }^{41} \mathrm{~V}$. Kaufman. Wavelengths and Energy Levels of Neutral $\mathrm{Kr}^{84}$ and Level Shifts in All Kr Even Isotopes. J. Res. Natl. Inst. Stand. Technol., 98, 1993. ${ }^{42}$ C. J. Humphreys and E. Paul Jr. Interferometric Observations in the Spectra of ${ }^{86}$ Kr. J. Opt. Soc. Am., 60:200-205, 1970.

${ }^{43}$ M. Martinez-Sanchez, J. Navarro-Cavallé, and E. Ahedo. Electron cooling and finite potential drop in a magnetized plasma expansion. Physics of Plasmas, 22(5):1-12, 2015.

${ }^{44} \mathrm{~K}$. Takahashi and A. Ando. Enhancement of axial momentum lost to the radial wall by the upstream magnetic field in a helicon source. Plasma Physics and Controlled Fusion, 59(5), 2017.

${ }^{45}$ I. D. Sudit and F. F. Chen. Discharge equilibrium of a helicon plasma. Plasma Sources Science and Technology, 5(1):43-53, 1996.

${ }^{46} \mathrm{D}$. Bose, T. R. Govidan, and M. Meyyappan. Modeling of a helicon plasma source. IEEE TRANSACTIONS ON PLASMA SCIENCE, , 31(4), 2003.

${ }^{47}$ F. F. Chen and D. Arnush. Generalized theory of helicon waves. I. Normal modes. Physics of Plasmas, 4(9):3411-3421, 1997.

${ }^{48}$ P. Chabert and N. Braithwaite. Physics of Radio-Frequency Plasmas. Cambridge University Press, 2011

${ }^{49}$ Yuriko TANIDA, Daisuke KUWAHARA, and Shunjiro SHINOHARA. Spatial Profile of Ion Velocity Distribution Function in Helicon HighDensity Plasma by Laser Induced Fluorescence Method. Transactions of the Japan Society for Aeronautical and Space Sciences, Aerospace Technology Japan, 14(ists30): $\mathrm{Pb}_{7}--P b_{1} 2,2016$

${ }^{50}$ S. A. Andersen, V. O. Jensen, P. Nielsen, and N. D'Angelo. Continuous supersonic plasma wind tunnel. Physics of Fluids, 12(3):557-560, 1969.

${ }_{81}^{51}$ E. Ahedo and M. Merino. Two-dimensional supersonic plasma acceleration in a magnetic nozzle. Physics of Plasmas, 17(7):1-15, 2010.

${ }^{52} \mathrm{M}$. Merino and E. Ahedo. Plasma detachment in a propulsive magnetic nozzle via ion demagnetization. Plasma Sources Science and Technology, 23(3), 2014

${ }^{53}$ E. Ahedo, S. Correyero, J. Navarro, and M. Merino. Macroscopic and parametric study of a kinetic plasma expansion in a paraxial magnetic nozzle. Plasma Sources Science and Technology1, 29(045017):0-20, 2020.

${ }^{54}$ Sara Correyero, Julien Jarrige, Denis Packan, and Eduardo Ahedo. Ion acceleration in the magnetic nozzle of an ECR thruster: Comparison of experimental measurements with a quasi 1D kinetic model. Space Propulsion 2018, (May):1-8, 2018 

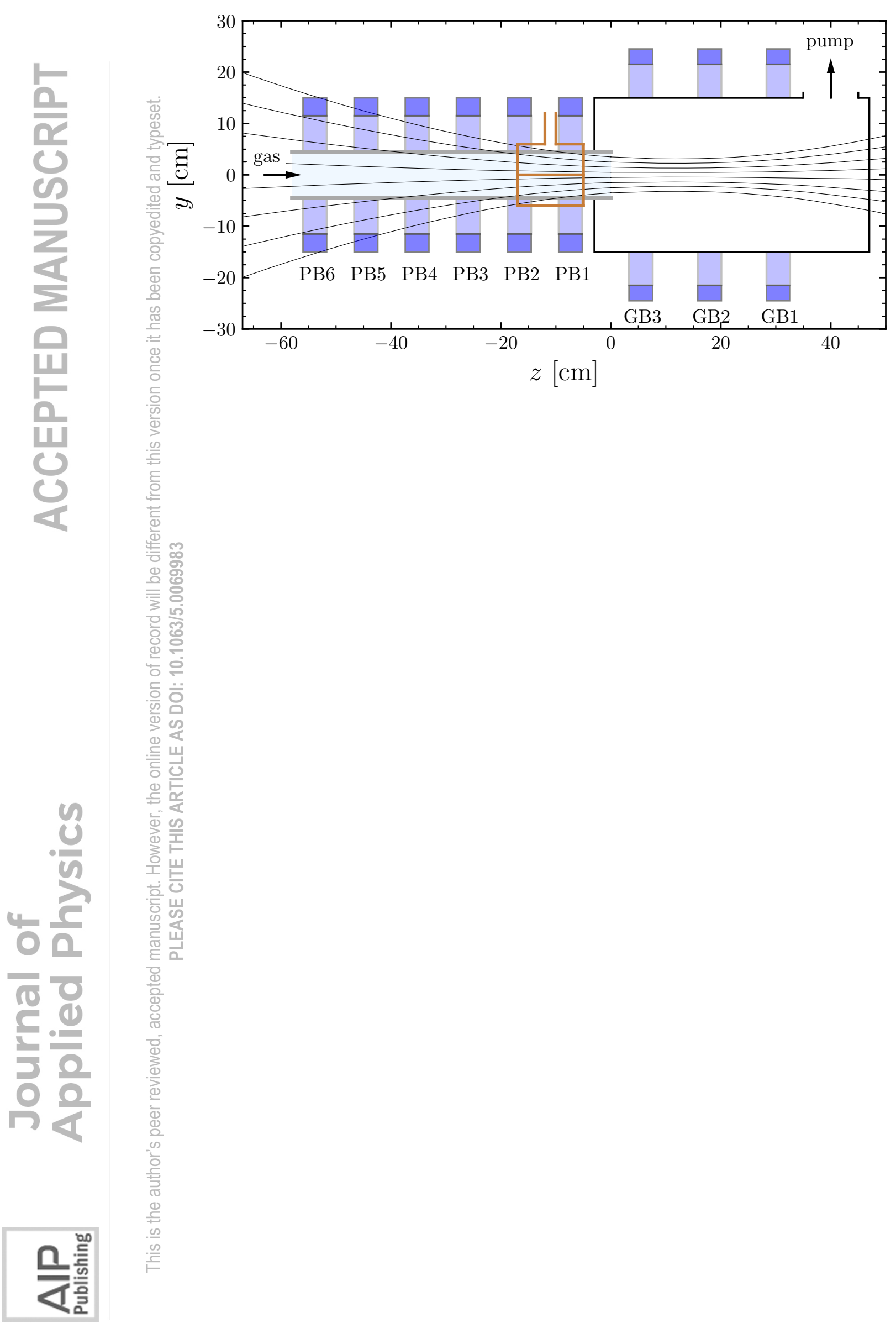


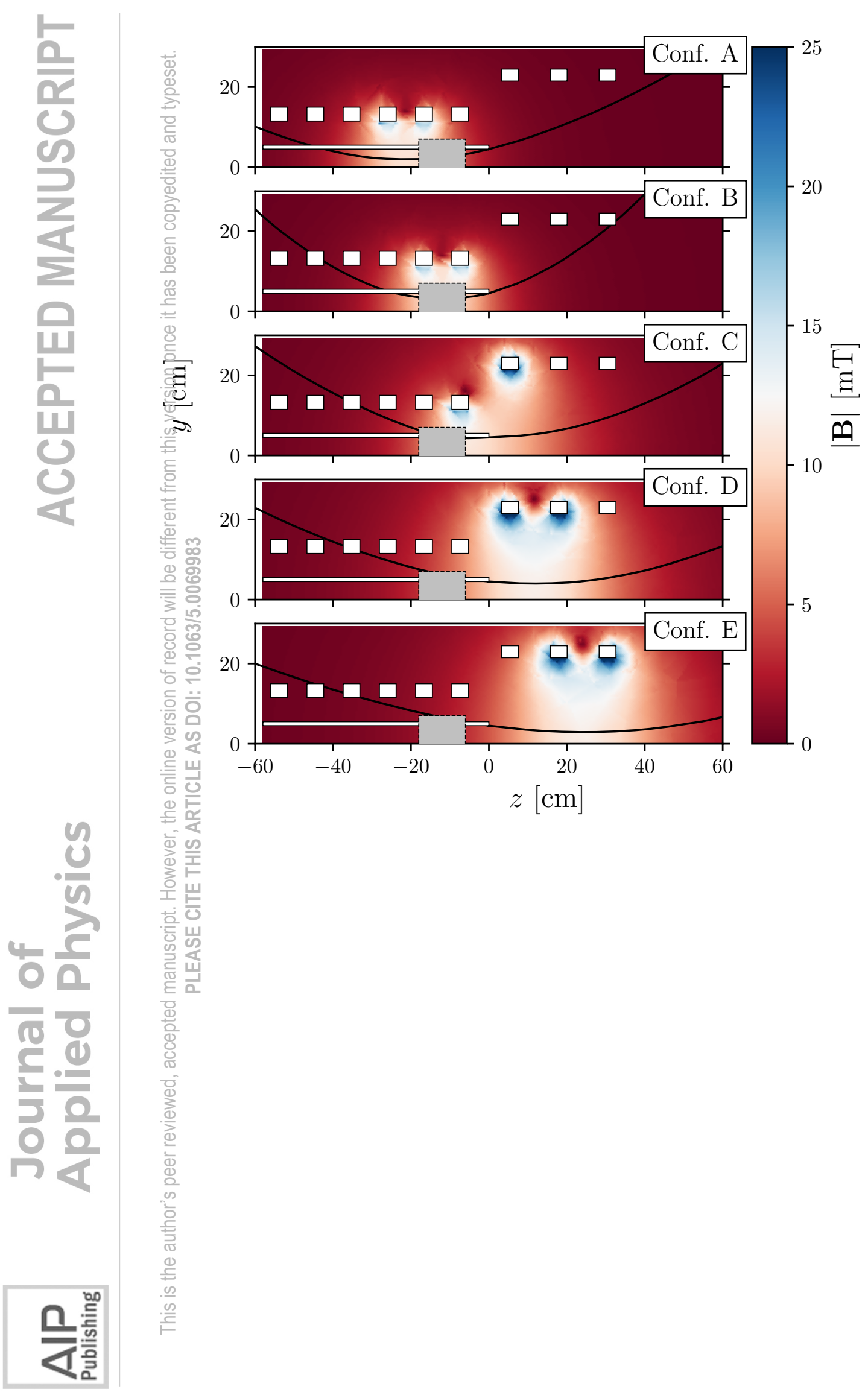




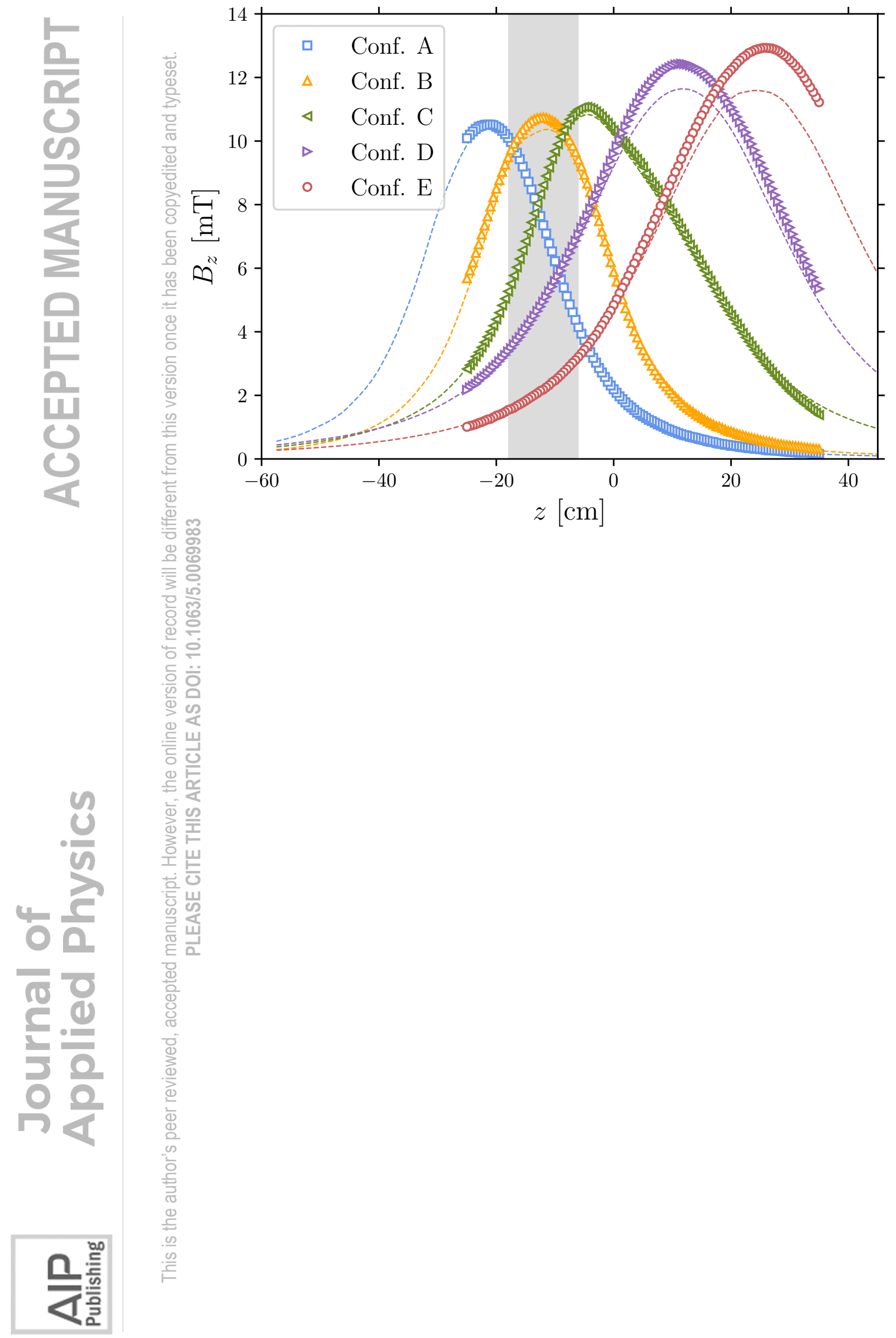


10 की 

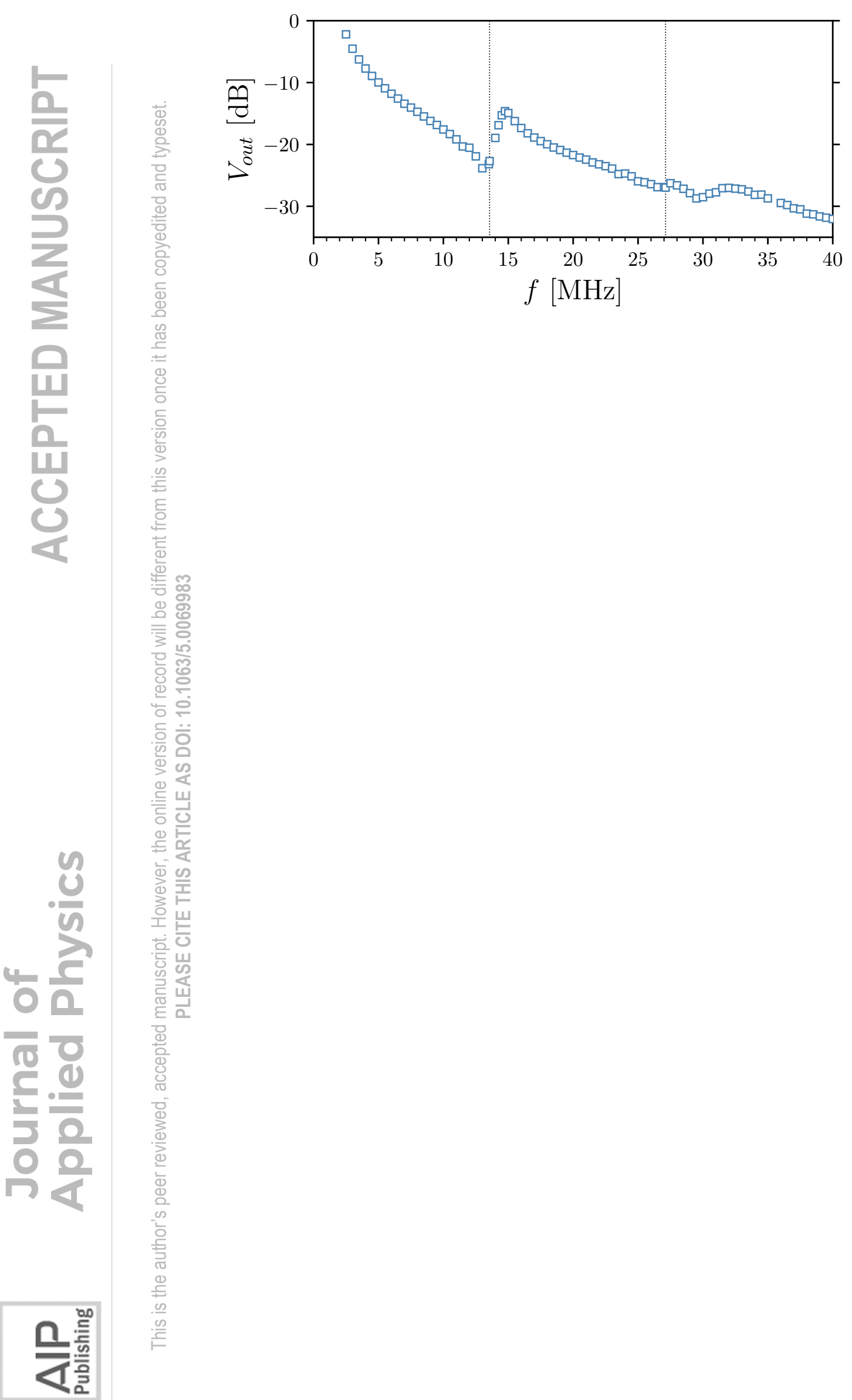

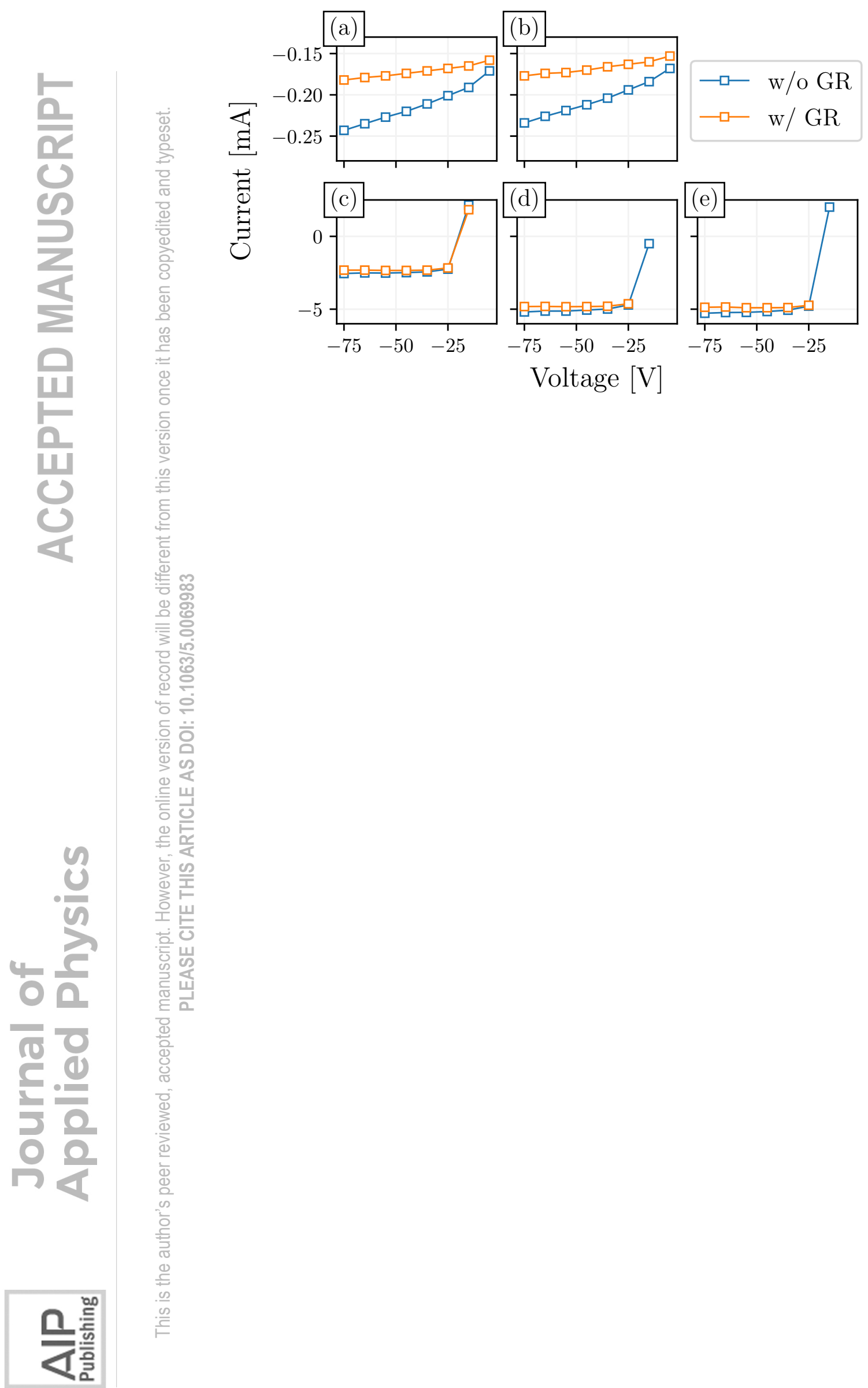

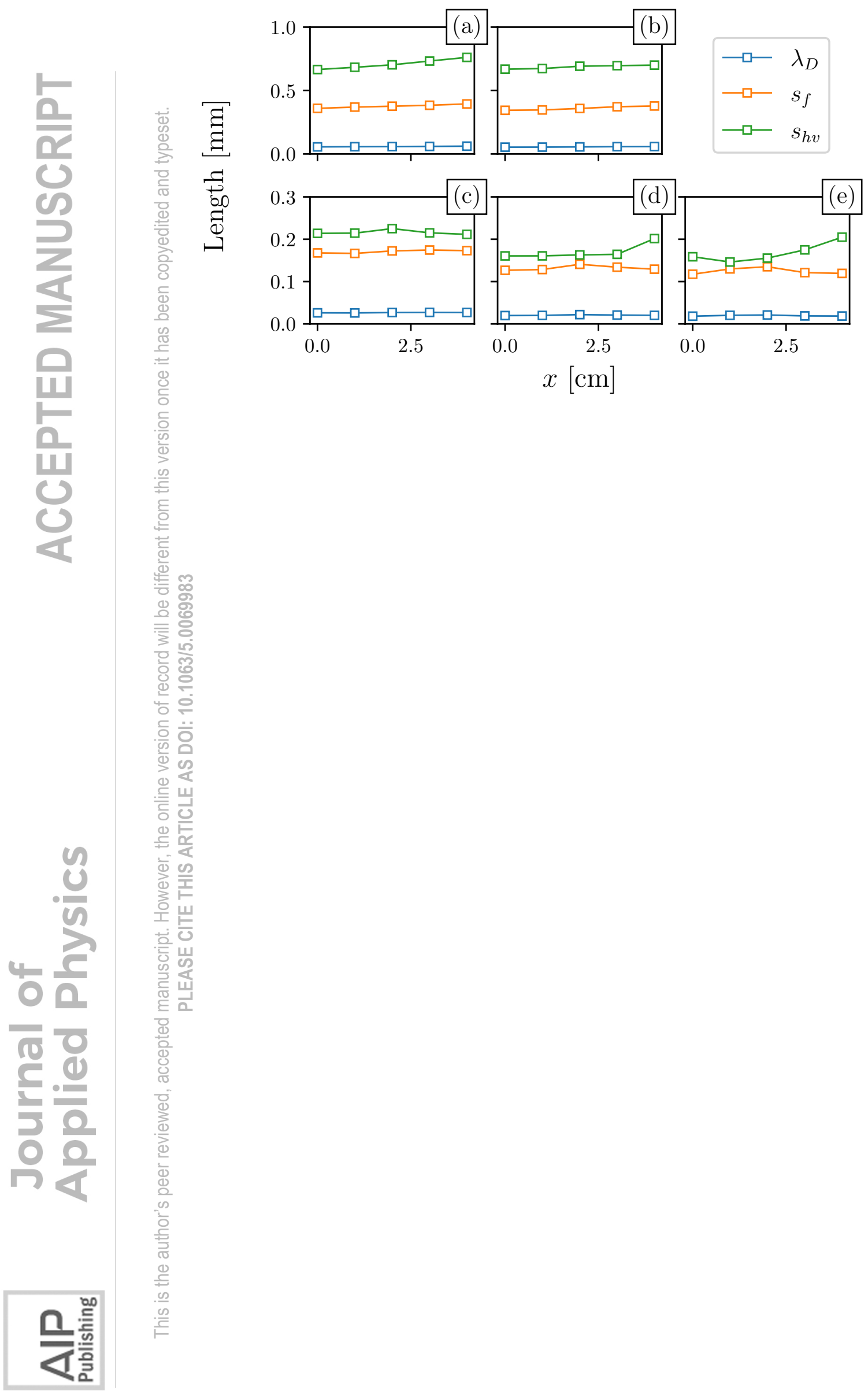


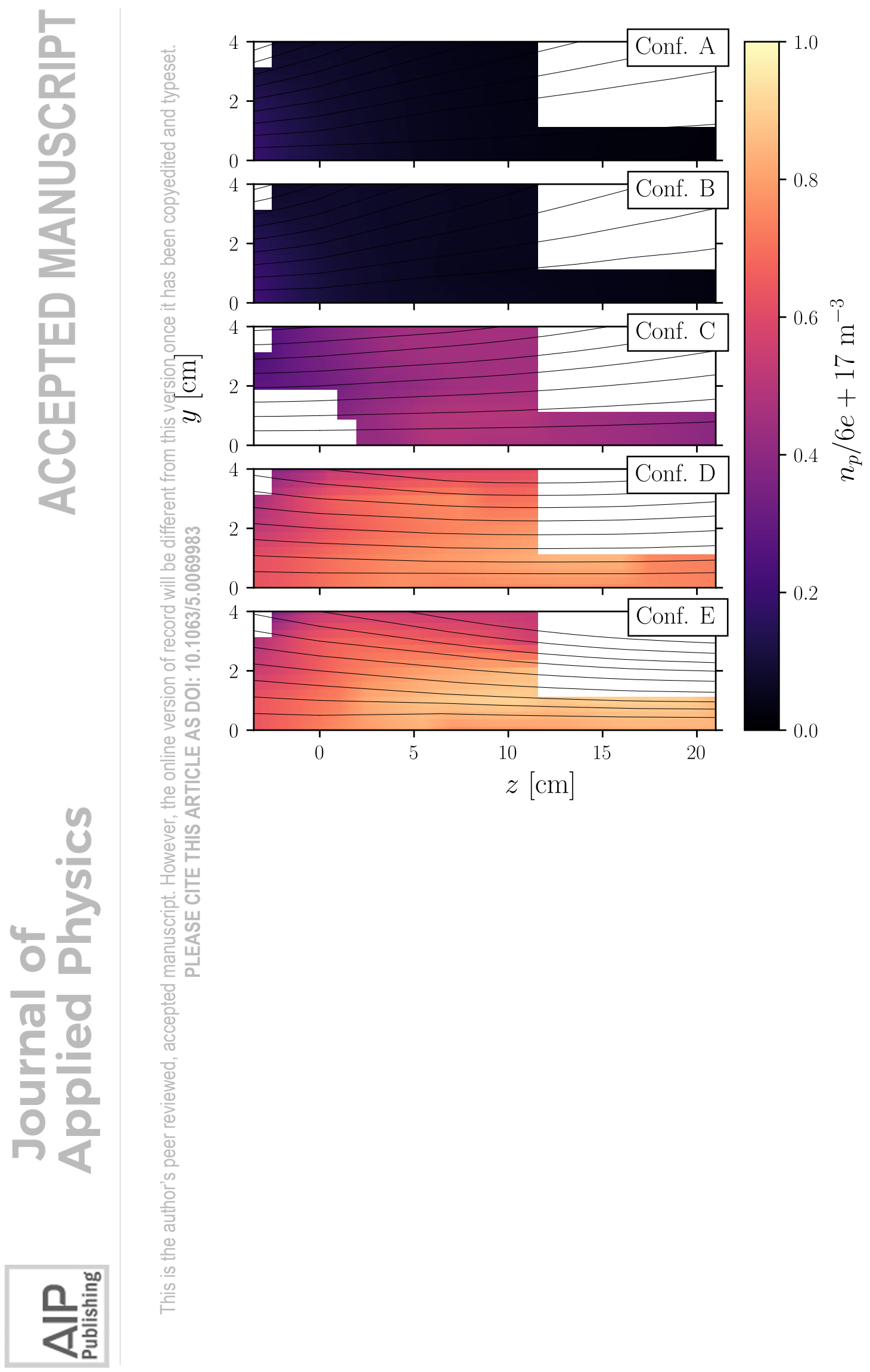




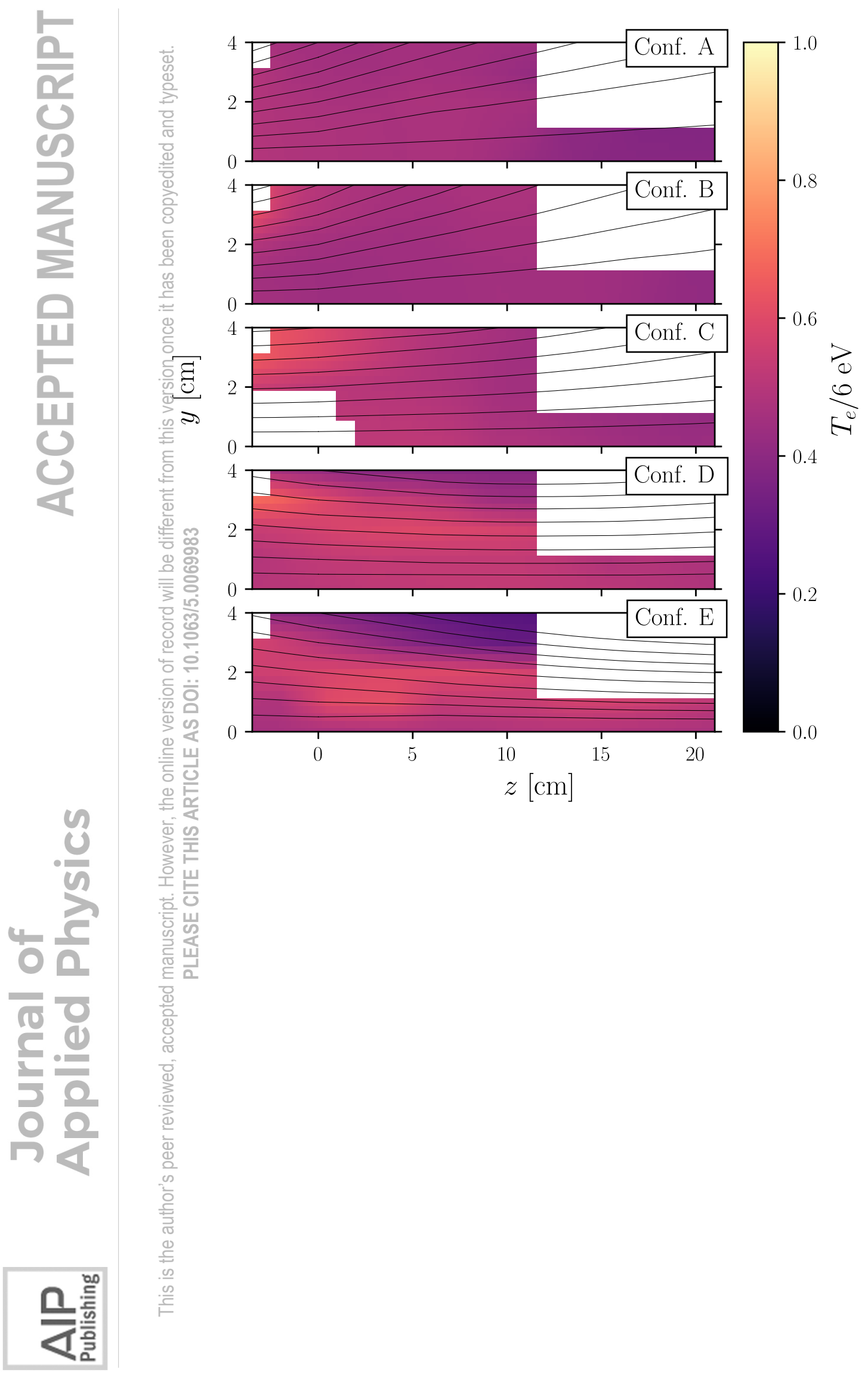




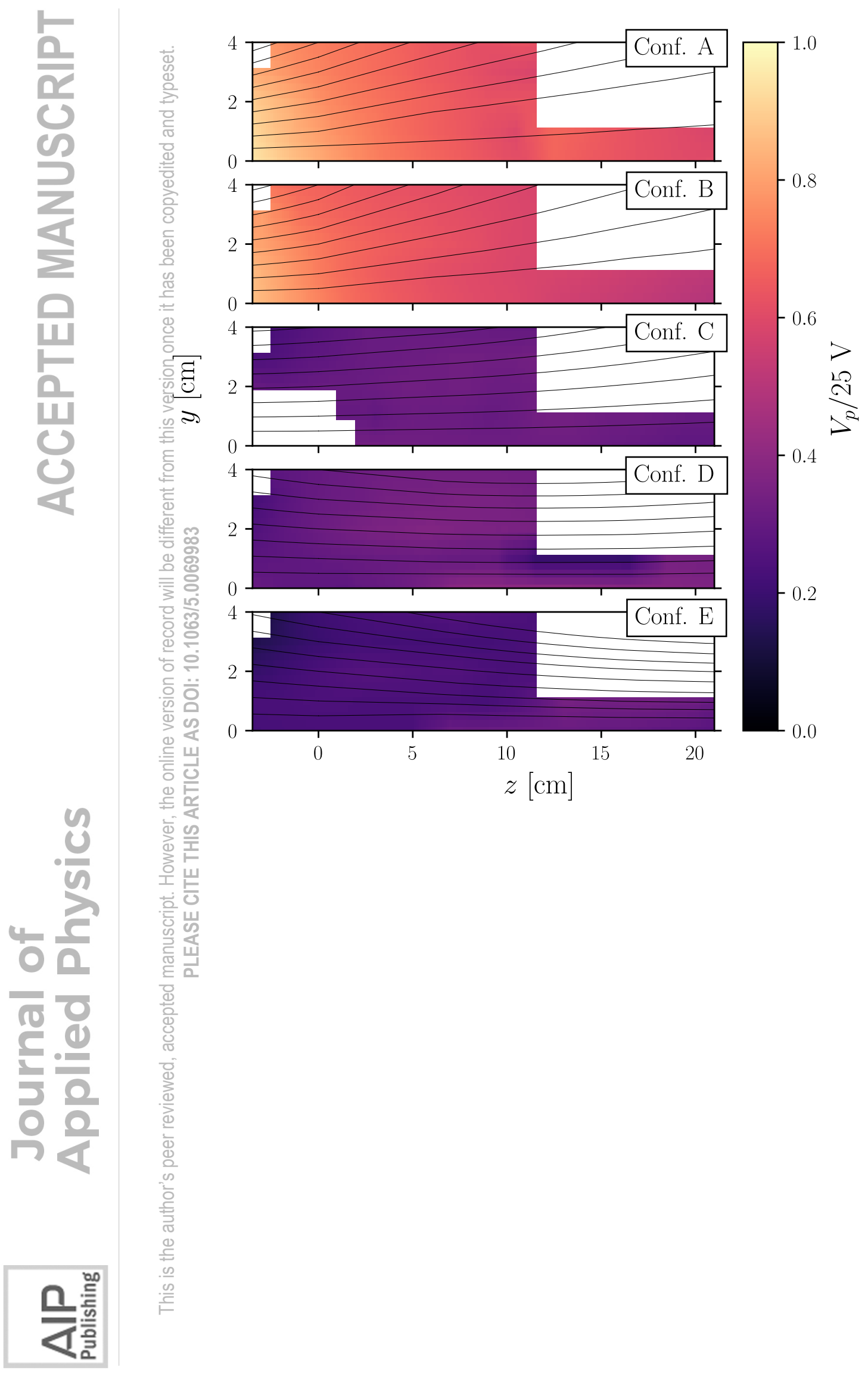




$\square \mathrm{A} \longrightarrow \mathrm{B} \longrightarrow \mathrm{C} \longrightarrow \mathrm{D} \longrightarrow \mathrm{E}$
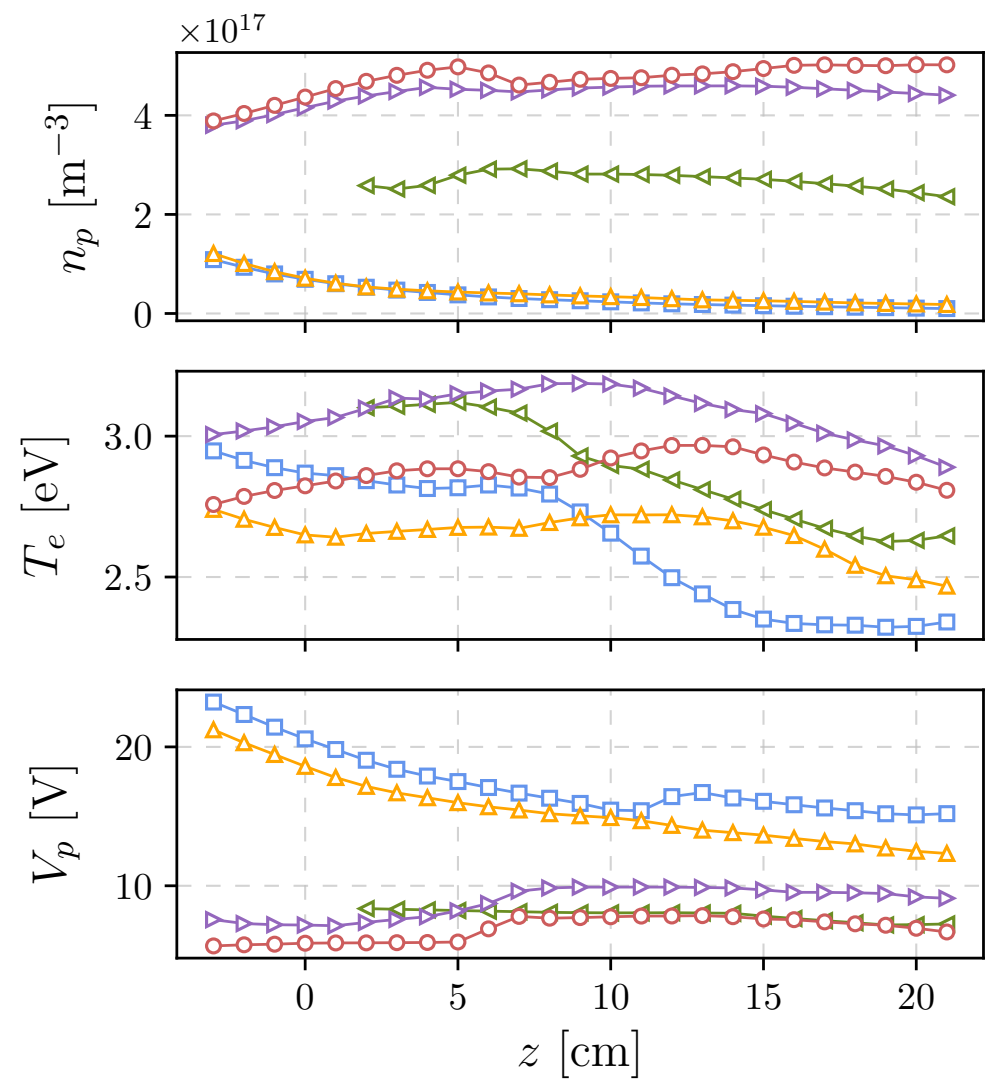


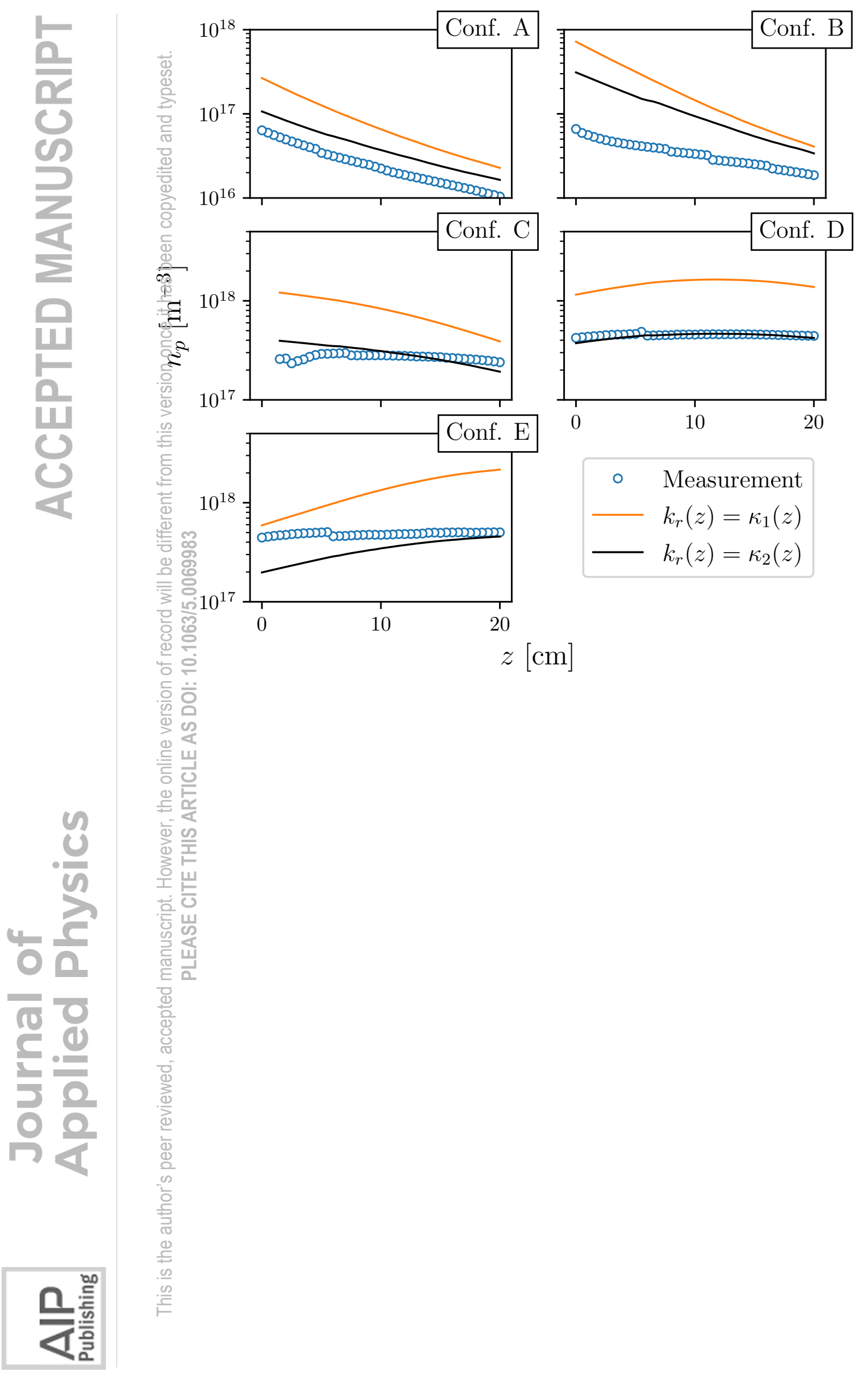




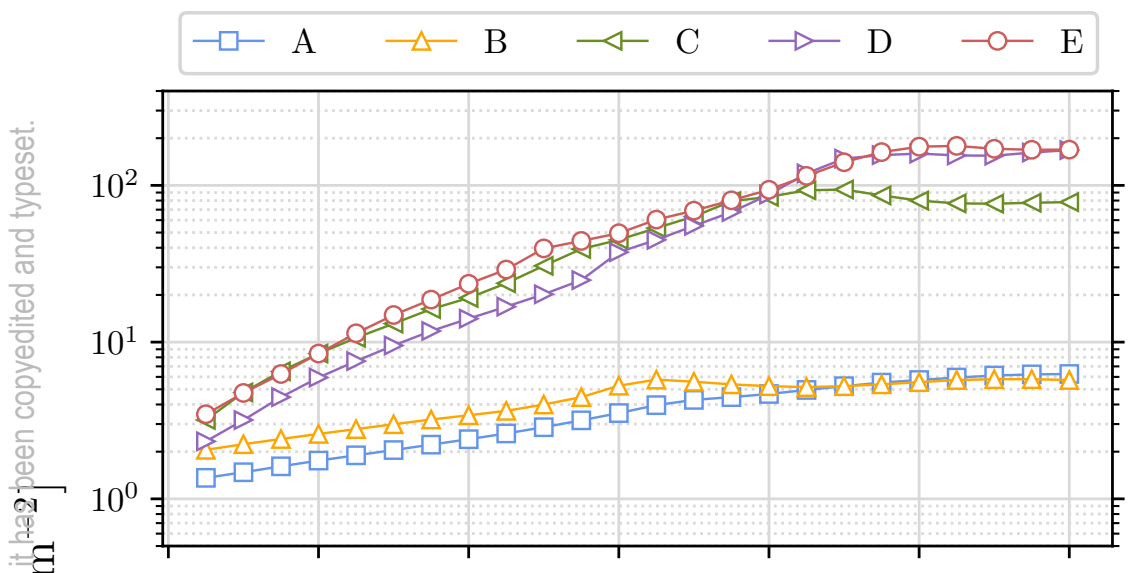

(a)

(b)
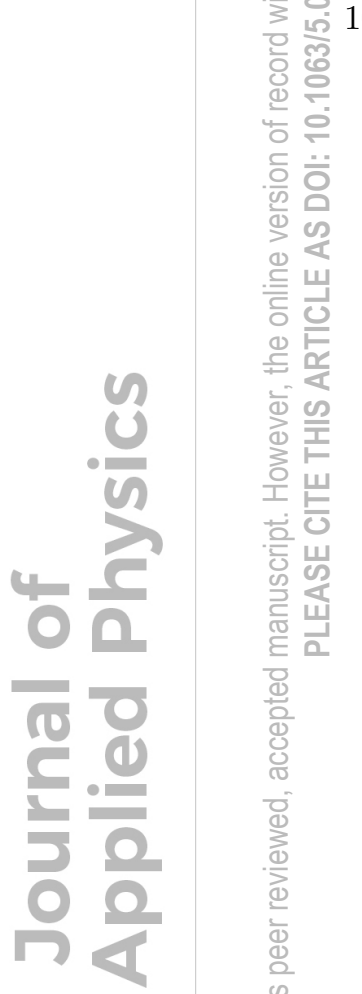

$0^{0} \mathrm{f} \begin{gathered}\Delta \Delta \Delta-\Delta \Delta \Delta \Delta \Delta \Delta \Delta \Delta \Delta \Delta \Delta \Delta \Delta \Delta \Delta \Delta \Delta-\Delta \cdots \\ \square-\square-\square-\square-\square-\square-\square-\square-\square-\square-\square-\square-\square-\square-\square-\square-\square-\square-\square-\square-\square-\square-\square-\square\end{gathered}$

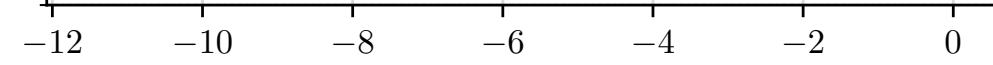

$x[\mathrm{~cm}]$

등

๘

(1)

플

등 븐

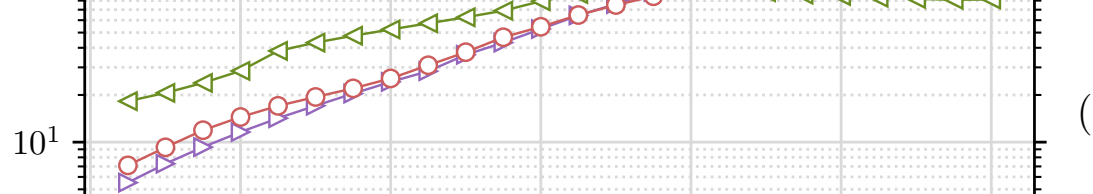




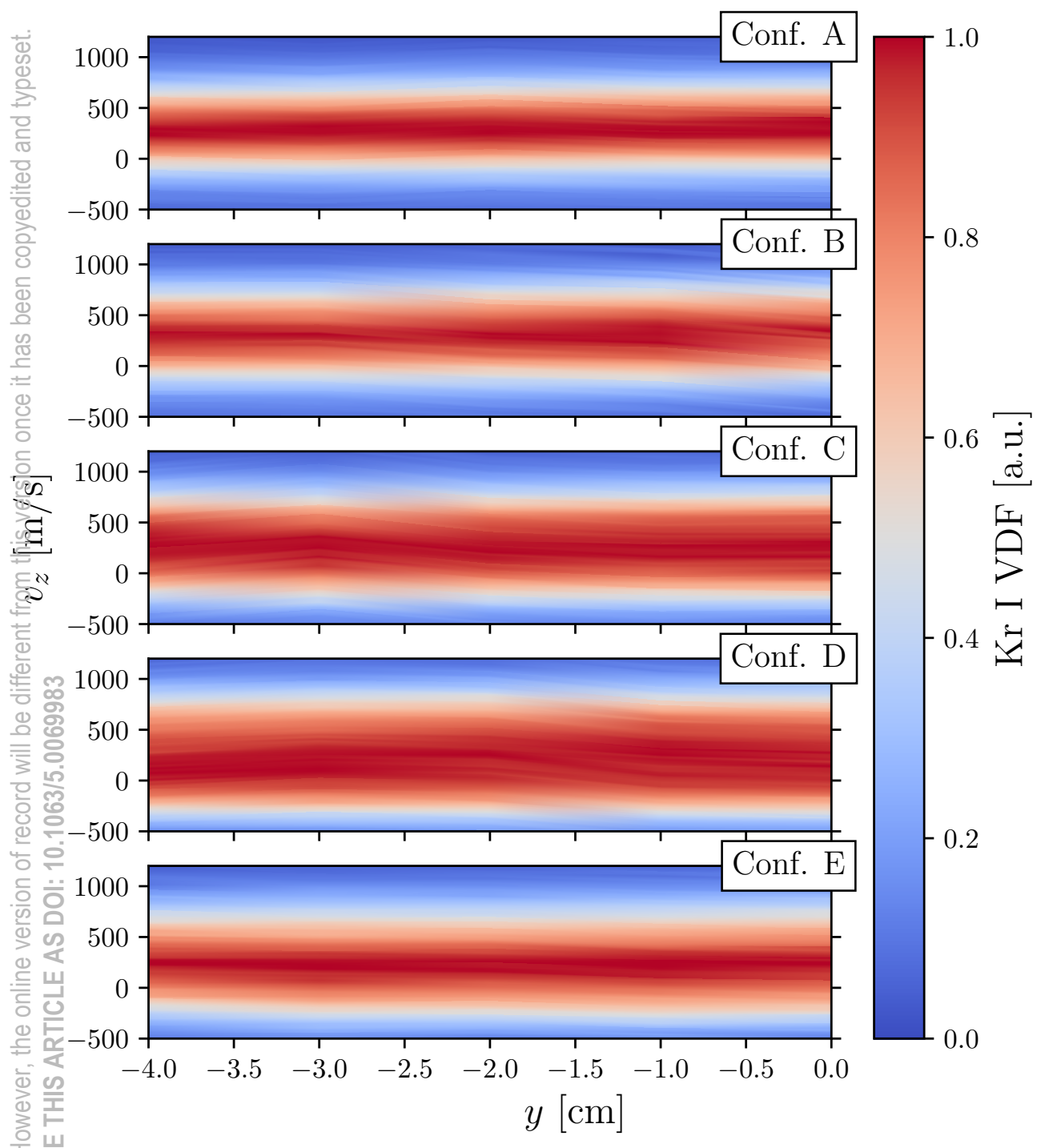




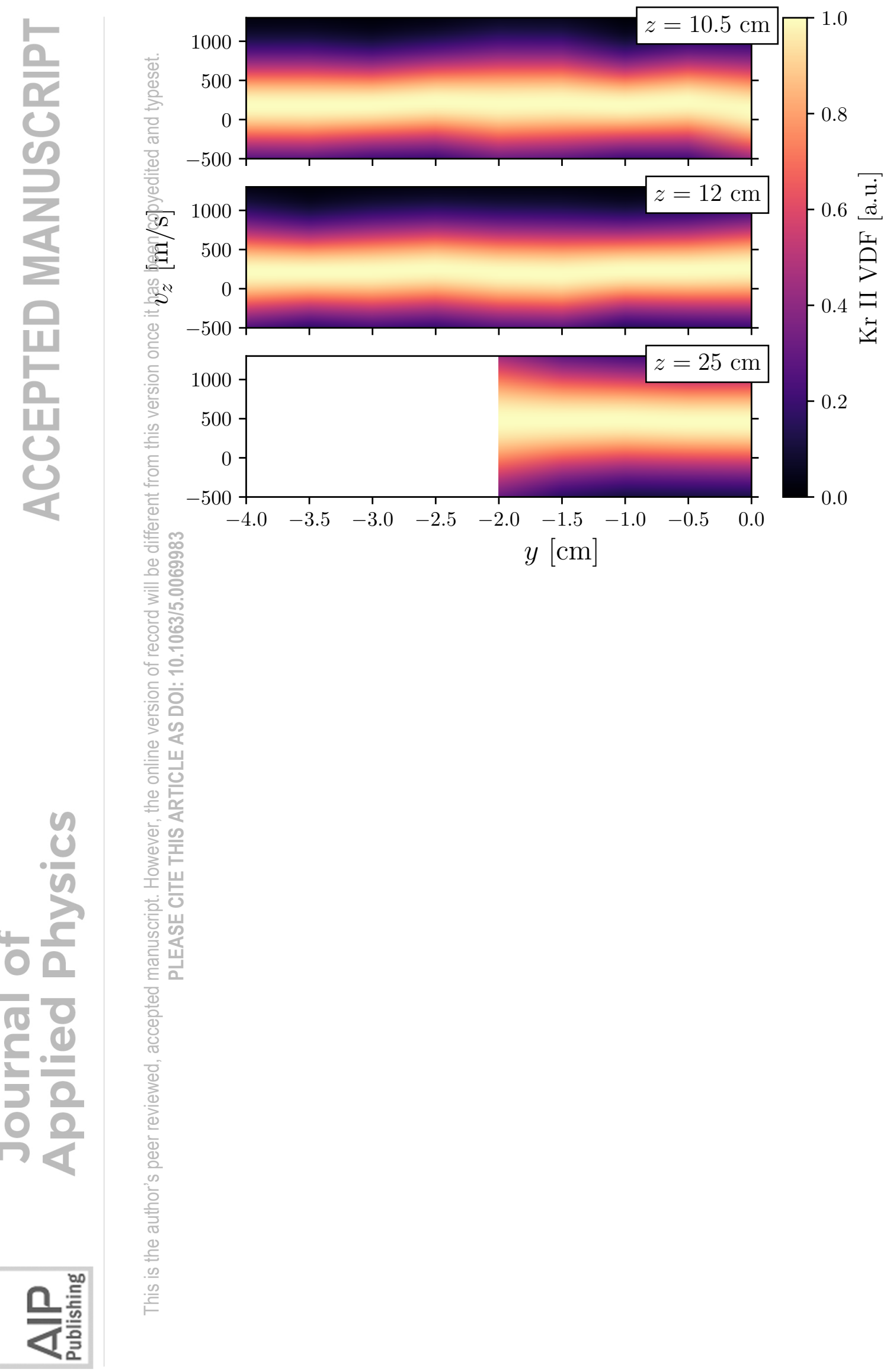




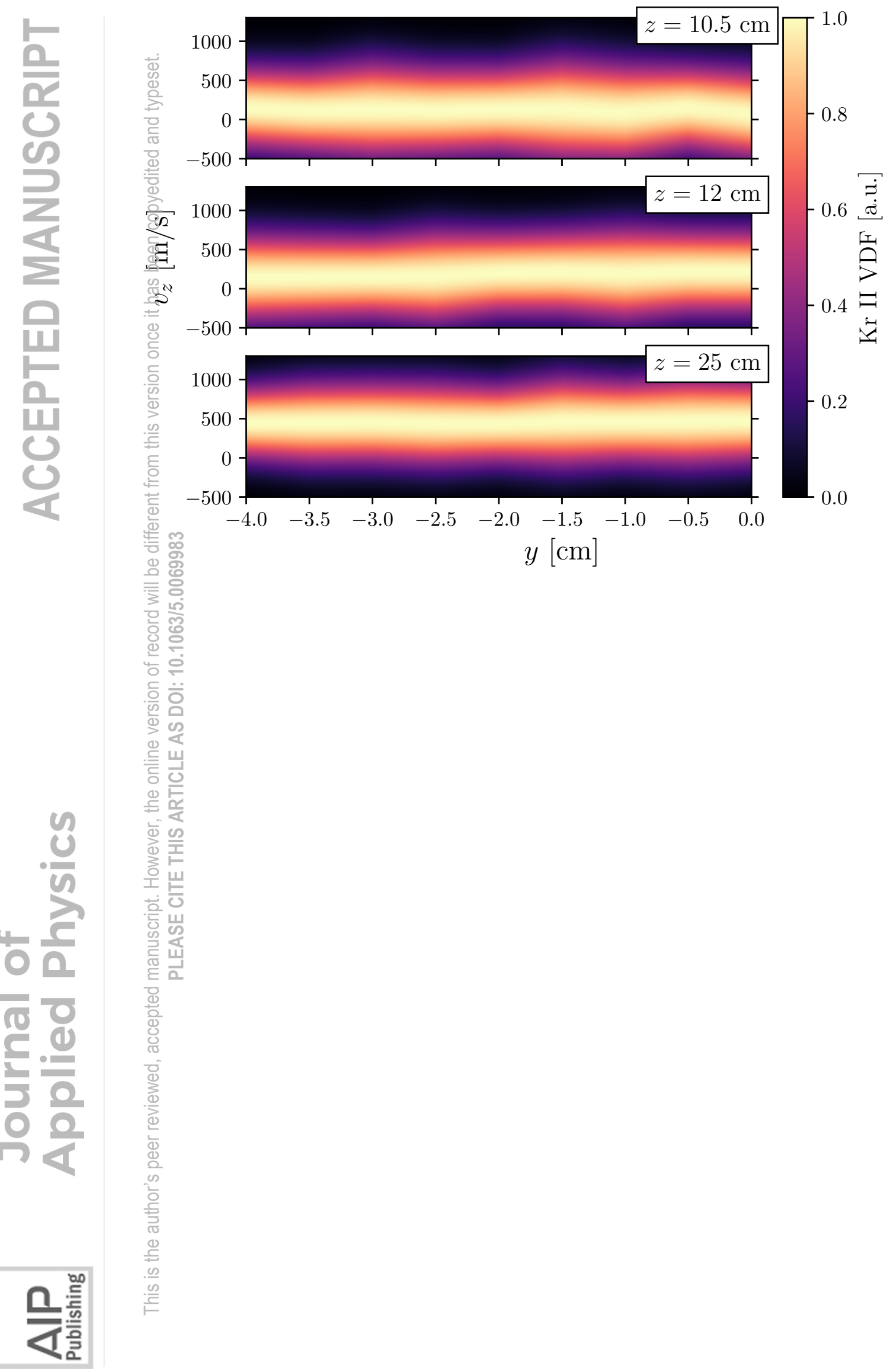




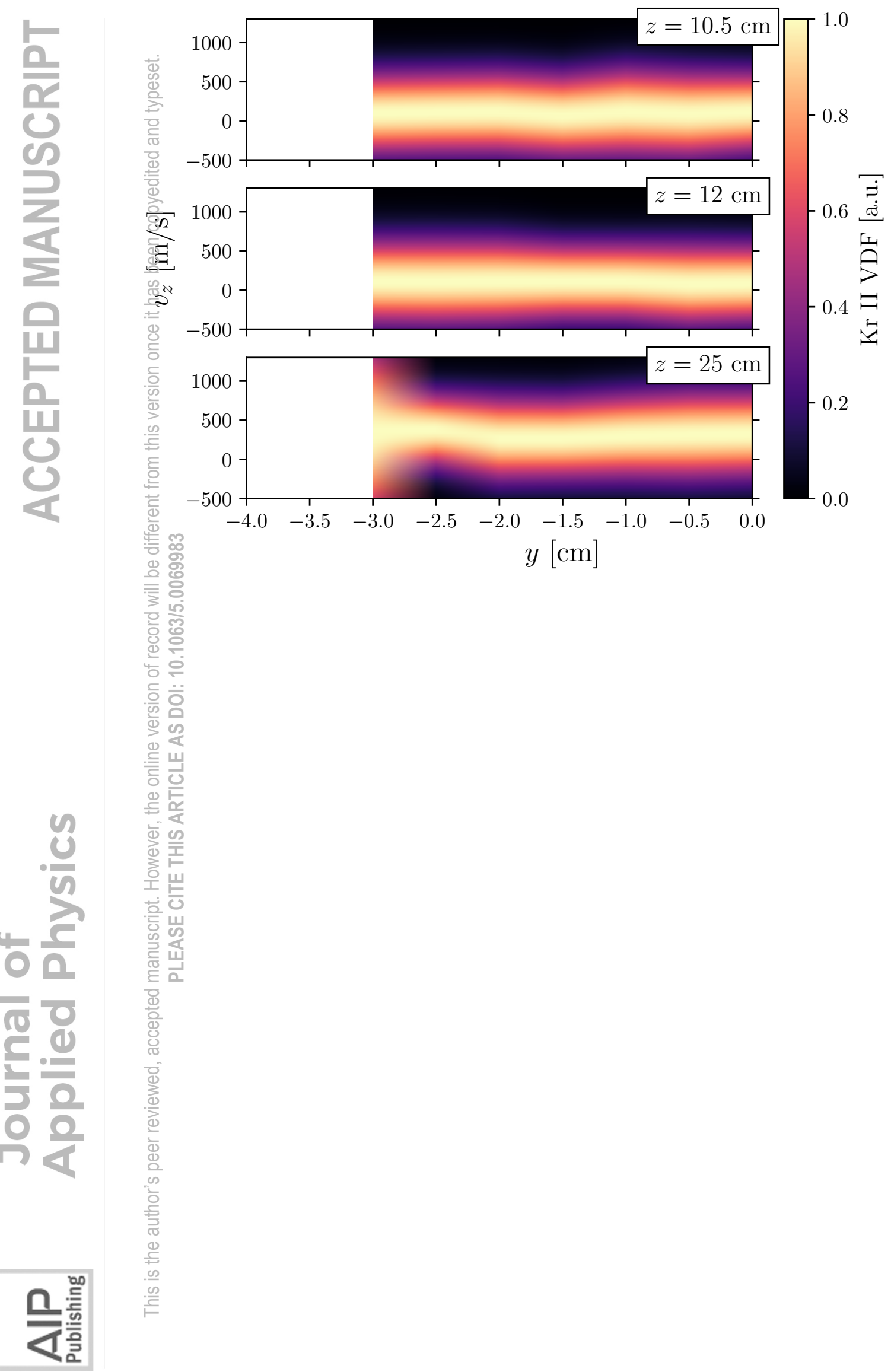




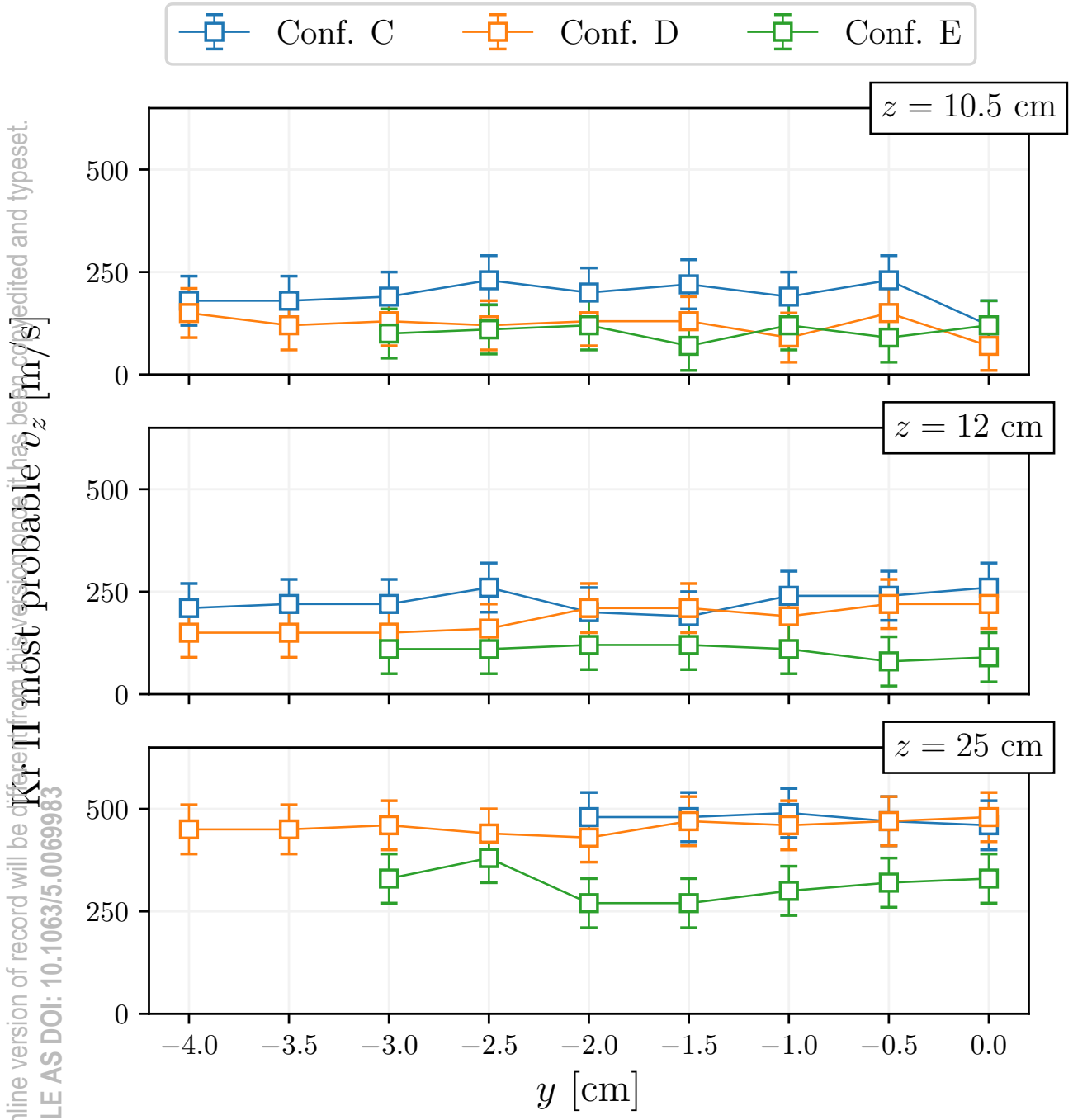




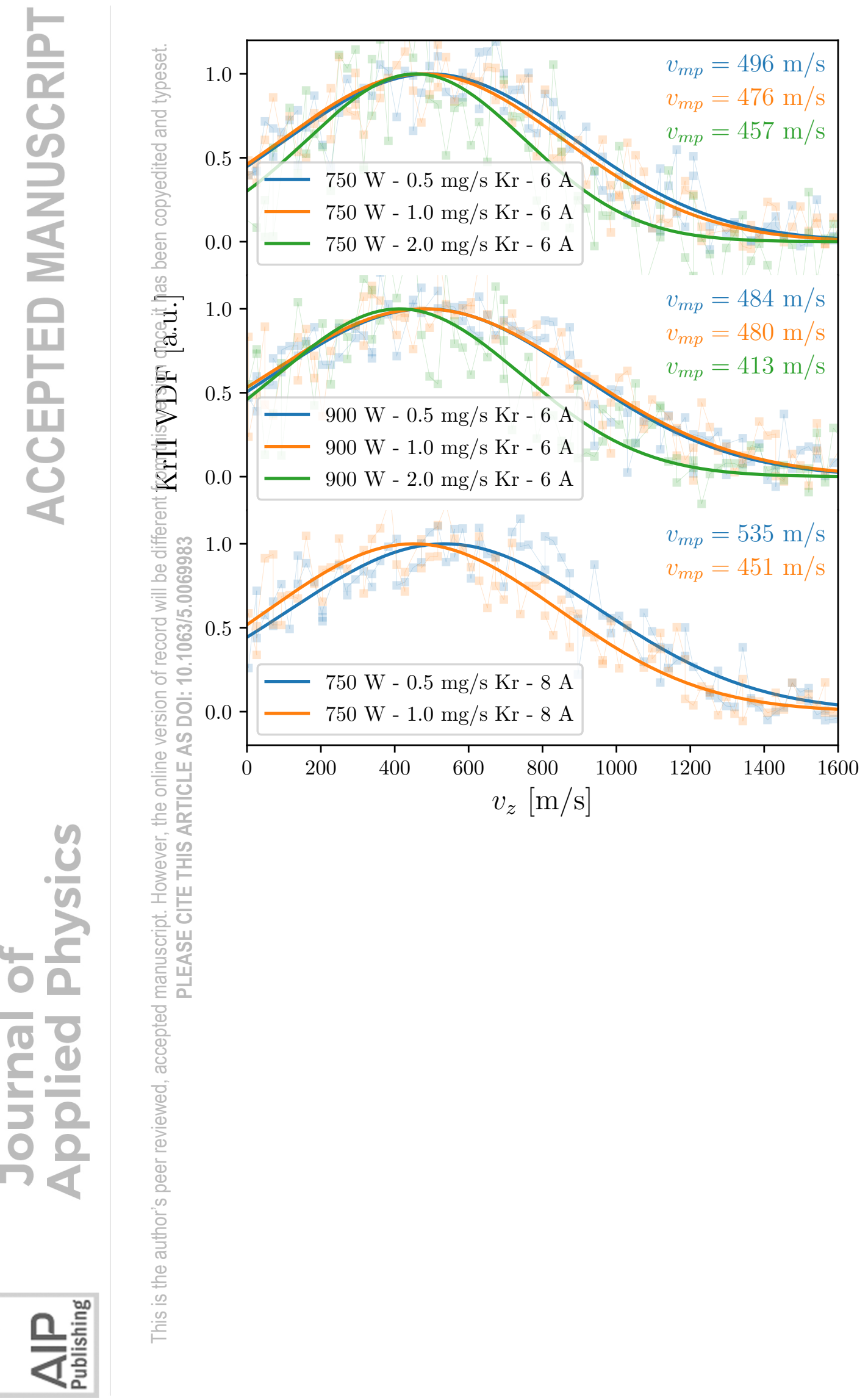

